\title{
Kapitel 3. \\ Mythos Ampelos - eine Erfindung des Nonnos?
}

\author{
Ever new magic! \\ Hast thou then lured hither, \\ Wonderful Goddess, by thy art, \\ The young, languid-eyed Ampelus, \\ Iacchus' darling - \\ Or some youth beloved of Pan, \\ Of Pan and the Nymphs? \\ That he sits, bending downward \\ His white, delicate neck \\ To the ivy-wreathed marge \\ Of thy cup; the bright, glancing vine-leaves \\ That crown his hair, \\ Falling forward, mingling \\ With the dark ivy-plants - \\ His fawn-skin, half untied, \\ Smear'd with red wine-stains? Who is he, \\ That he sits, overweigh'd \\ By fumes of wine and sleep, \\ So late, in thy portico? \\ What youth, Goddess, - what guest \\ Of Gods or mortals?
}

Matthew Arnold ${ }^{1}$

\subsection{Ampelos in der antiken Literatur}

Mythen zum Wein und zu seiner Entstehung stellen eine Tradition dar, die bis in das Alte Testament zurückverfolgt werden kann. Dort pflanzt Noah nach der Sintflut als Erster den Wein, wird als Erster trunken und von seinem Sohn Ham entblößt in seinem Zelt entdeckt ( $L X X$ Ge. 9,20 - 27). ${ }^{2}$ Überlieferungen über die Entstehung, Kultivierung und (bisweilen folgenschwere) Konsumierung des Weines werden auch in der Mythologie der Griechen und Römer reflektiert, insbesondere in der Dionysos-BacchusFigur, die Pflanze und Getränk unter allen Menschen der Oikumene verbreitet. Spuren von Aitien für Weinstock und Wein finden sich in der antiken Literatur zunächst jedoch nicht in Zusammenhang mit Dionysos: Hekataios von Milet berichtet - Athenaios

1 The Strayed Reveller, in: Arnold (1849) $15 \mathrm{f}$.

2 Vgl. Otto (2011) 136f.; Anagnostakis u. Papamastorakis (2004); Pitte (2009) 42 - 46. 


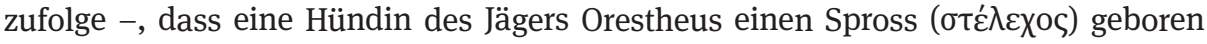
habe, der, in der Erde vergraben, den ersten Weinstock habe erwachsen lassen. ${ }^{3}$

Mit seiner Erzählung über die Genese der Weinrebe schließt Nonnos an ebendiese Traditionslinie an und ist einem kulturellen Erbe verpflichtet, dessen Bedeutsamkeit zu allen Zeiten für die antiken Kulturen nicht überschätzt werden kann: dem dionysischen Sagenkreis. Die Motive Tod und Wiederauferstehung des Weingottes sind aus dem orphischen Mythos um das Leben und Sterben des Zagreus bekannt (Nonn. $D$. 6,163-205). ${ }^{4}$ An die Episode vom gewaltsamen Tod des ersten Dionysos durch die Titanen schließt die Geschichte von den drei Göttergenerationen an: Die Göttertrias, Zagreus, Dionysos und Iakchos, repräsentiert in ihrer chronologischen Abfolge das bakchische Prinzip; mit dem Tod des Zagreus, seiner Ermordung mit dem Ziel der Bestrafung der Titanen und der von Zeus veranlassten Auslöschung des gesamten bisherigen Lebens durch Weltenbrand und Sintflut geht ein Weltzeitalter zugrunde. Nonnos bedient sich offenkundig orphischer Überlieferungen vom Sterben und von der Wiedergeburt der Gottheit (vgl. Orph. Fr. 214 Kern). ${ }^{5}$ Diese Tradition nimmt auch einen festen Platz in der spätantiken Literatur ein, so wird sie etwa bei Himerios als Vergleichsmythos verwendet (4. Jh. n. Chr.): In der 45. Rede, die die Gesundung eines Schülers zum Thema hat, kommt Himerios auch auf den durch die Telchinen oder Titanen verursachten Tod des Dionysos sowie auf dessen Wiedererweckung durch Zeus zu sprechen. ${ }^{6}$ Noch bevor sich das Schicksal des Dionysos zum Guten wendet, wird die

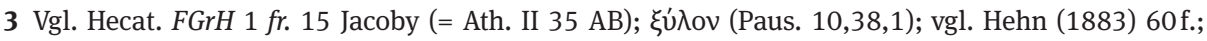
Kerényi (1994) 61f.; zu Orestheus, dem Sohn des Deukalion, siehe RE XVIII/1, 1016.

4 Zum Mythos des Zagreus und zu seiner Funktion in den Dionysiaka vgl. D’Ippolito (1964) 216 - 227; Hernández de la Fuente (2002); Hernández de la Fuente (2008) 192 - 201; García-Gasco (2011); zum Einfluss der orphischen Dichtungen auf Nonnos siehe Livrea (2014) und Otlewska-Jung (2014); vgl. außerdem Kerényi (1994) 63 - 68. - Zum Einfluss der Orphik in der Spätantike vgl. Graf (1993); Guettel Cole (1993); Herrero de Jáuregui (2010); Herrero de Jáuregui (2010a); Herrero de Jáuregui u. a. (2011). 5 Einer antiken Interpretation zufolge ist der orphische Zagreus-Mythos eng mit dem Weingott Dionysos verbunden, demnach entspreche die Zerstückelung des Zagreus dem Pflücken der Trauben bei der Weinlese und die Wiedergeburt des Gottes der Gewinnung des Getränks; vgl. West (1983) 141. 6 Neben der Geschichte um Dionysos Zagreus nennt Himerios auch den Zug des Gottes nach Indien (Him. Or. 18,2) und dessen Geburt (Him. Or. 27), er äußert sich zum Eigennamen Bakchos (Him. Or. 44,3) und verweist auch auf dessen Jugendgeschichte (Him. Or. 47,6; 68,5). Ferner thematisiert er

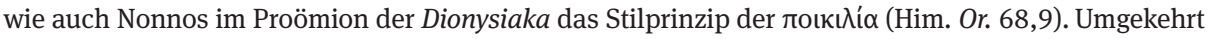
wendet Nonnos in den Dionysiaka ein vergleichbares rhetorisches Instrumentarium wie in den Reden des Himerios an, vgl. etwa Him. Or. 8, die Trauerrede auf Himerios' verstorbenen Sohn Rufinus, die wie die beiden Trauerreden des Dionysos in der Ampelos-Episode typische Elemente der Epitaphien-Literatur aufweist: das Gebirge als Unglücksort, der allzu frühe Tod und die verpasste Hochzeit (Him. Or. 8,8) sowie die durch den Vater angestrebte Unsterblichkeit des Sohnes (Him. Or. 8,23). Beide, Himerios und Nonnos, schöpfen aus einem gemeinsamen kulturellen Erbe, das nicht nur pagane

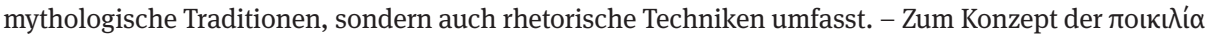
in der Rhetorik siehe Heath (1989) 95 Anm. 9; zum Einfluss der Rhetorik auf Nonnos siehe Kap. 6. 
Trauer des verstorbenen Gottes auf besondere Weise zum Ausdruck gebracht (Him. Or. 45,4): ${ }^{7}$

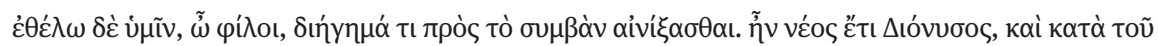

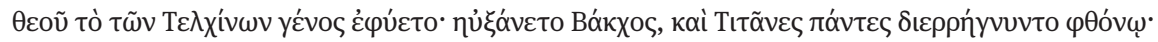

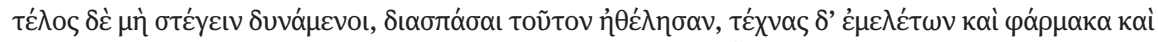

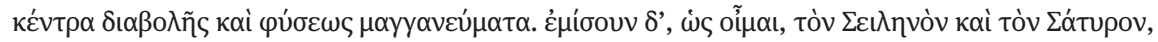

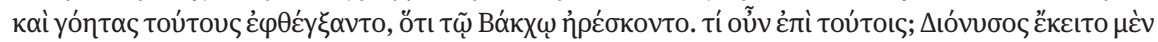

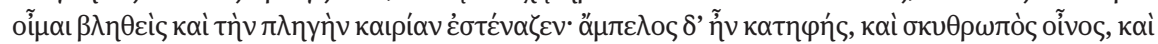

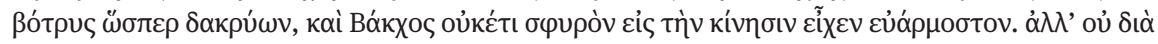

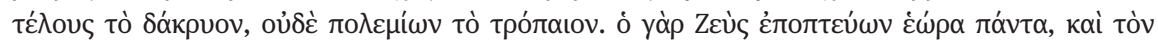

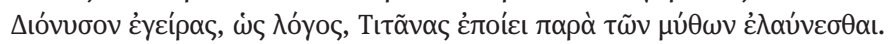

Ich will euch, meine Freunde, eine Geschichte zu dem, was uns widerfahren ist, in knappen Worten skizzieren: Dionysos war noch jung, und es erhob sich das Geschlecht der Telchinen gegen den Gott. Er wuchs und alle platzten vor Neid. Schließlich konnten sie dies nicht verbergen und wollten ihn in Stücke reißen, sie wandten ihre Fertigkeit an, bereiteten Gift, setzten den Stachel der Verleumdung ein und die ihnen angeborenen Tricks. Sie haßten meiner Meinung nach den Silen und den Satyr, und nannten diese Betrüger, weil sie bei Bakchos angesehen waren. Was geschah nun infolgedessen? Dionysos lag getroffen da und beklagte den Todesstreich. Er war nun ein in Trauer welk herunterhängender Weinstock, und der Wein war dunkel und trüb, die Weintraube war wie eine Träne, und Bakchos konnte sich nicht mehr mit seinen wohlgeformten Füßen bewegen. Aber die Träne floß nicht für immer, auch der Sieg der Feinde war nicht von Bestand. Denn Zeus, der schützend herabsah, sah alles und, nachdem er Dionysos wieder zum Leben erweckt hatte, wie erzählt wird, ließ er gemäß der mythologischen Darstellungen die Titanen verjagen.

Dionysos und sein charakteristisches Attribut, der Wein, werden in der Übersetzung Völkers einander angeglichen, ja Dionysos scheint hier geradezu eine Metamorphose in einen Weinstock vollzogen $\mathrm{zu}$ haben (,Er war nun ein in Trauer welk herunterhängender Weinstock [...]“), die Szenerie scheint an die von Nonnos geschilderte Metamorphose des Satyrn in die Pflanze zu erinnern. Dass dies nicht notwendigerweise vorliegen muss, zeigt Robert Penellas Übersetzung: „Dionysus lay wounded, I think, and bemoaned the serious blow he had suffered. The vine was dejected, wine was sad, grapes seemed to be crying, and Bacchus's ankle was not yet in any condition to move. "8 Illustriert wird die Trauer des Weinstocks, des Weines und der Trauben über Dionysos' Tod. Das Motiv der Trauer um einen Verstorbenen kehrt auch in den Dionysiaka wieder: An besonders prominenter Stelle in der Ampelos-Episode beweint nicht nur Dionysos, sondern auch die umgebende Natur den auf tragische Weise ums Leben Gekommenen (Nonn. D. 11,224-350; 12,117-137). ${ }^{9}$ Bei den Motiven des frühzeitigen und gewaltsamen Todes, der rituellen Klage und Wiederauferstehung, die sich sowohl bei Himerios als auch bei Nonnos finden, handelt es sich nicht um eine direkte Einflussnahme des Rhetorikers auf den Dichter, vielmehr schöpfen beide Autoren aus

7 Text: Colonna (1951) 184; Übersetzung: Völker (2003) 286f.; vgl. Koehler (1853) 25.

8 Penella (2007) 91.

$9 \mathrm{Zu}$ den Trauerreden des Dionysos siehe S. 126-131; vgl. auch die Trauer des Dionysos bei der Verwundung des Hymenaios (Nonn. D. 15,370-422). 
denselben mythisch-literarischen Traditionen um Leben, Sterben und Wiedergeburt des Weingottes.

Obwohl derartige mythische Traditionen um die Geburt des Weines im Hintergrund der Ampelos-Episode zu denken sind, bietet Nonnos dennoch eine in der antiken Literatur unbekannte Erzählung, wobei er sich insofern von allem Bestehenden löst, als er mit dem Schicksal des Ampelos einen völlig neuen Mythos schafft. Weder in der griechischen noch in der römischen Literatur sind zweifelsfreie Quellen, Vorlagen oder Entsprechungen belegt. Nur einige wenige singuläre Zeugnisse nennen Ampelos, das prominenteste davon liefert Ovid in den Fasten (Ov. fast. 3,403-414): ${ }^{10}$

Cum croceis rorare genis Tithonia coniunx coeperit et quintae tempora lucis aget,

sive est Arctophylax, sive est piger ille Bootes, 405 mergetur visus effugietque tuos.

at non effugiet Vindemitor: hoc quoque causam unde trahat sidus parva docere mora est.

Ampelon intonsum satyro nymphaque creatum fertur in Ismariis Bacchus amasse iugis.

tradidit huic vitem pendentem frondibus ulmi, quae nunc de pueri nomine nomen habet;

dum legit in ramo pictas temerarius uvas, decidit; amissum Liber in astra tulit.

Wenn die Gemahlin des Tithonos angefangen hat, von ihren Safranwangen Tau zu träufeln, und den fünften Tag beginnt, dann geht (ein Sternbild), sei es, daß es der Bärenwächter, sei es, daß es der langsame Bootes ist, im Meere unter und entzieht sich deinen Blicken. Nicht dagegen verschwindet der Winzer. Auch läßt sich in Kürze sagen, wo dies Sternbild seinen Ursprung hernimmt. Bacchus hat, so heißt es, auf den ismarischen Höhen Ampelos geliebt; der war ein Knabe, dessen Haar noch nicht geschoren war, und eine Nymphe und ein Satyr waren seine Eltern. Diesem gab er einen Weinstock, der im Laub der Ulme hing und jetzt von dem Namen dieses Knaben seinen Namen trägt. Als er auf einem Aste unvorsichtig bunte Trauben las, fiel er hinunter. Liber hob den Jüngling, den er (so) verloren hatte, zu den Sternen empor.

Ampelos wird nicht wie bei Nonnos das Opfer eines Stieres, sondern kommt durch den Sturz von einer Ulme zu Tode, an die Stelle der Metamorphose in den Weinstock tritt bei Ovid der Katasterismos zum Sternbild des Vindemitor. Eine Verbindung zum Weinbau und zum Wein besteht nur insofern, als Ampelos beim Pflücken von „gesprenkelten Weintrauben“ (pictas uvas [Ov. fast. 3,413]) stürzt.

In der griechischen Literatur findet sich außer in den Dionysiaka nur eine einzige weitere Stelle zu Ampelos, und zwar in den sog. Pseudo-Klementinen, einer romanhaften Erzählung über den Apostel Petrus und seinen Schüler Klemens, in der in einer Unterredung zwischen Klemens und Appion, einem Schüler des Häretikers Simon

10 Text und Übersetzung: Bömer (1957) 154f.; vgl. Koehler (1853) $24 \mathrm{f}$. - Zu den spärlichen literarischen Quellen für Ampelos vgl. Roscher I/1, 292; DKP 1, 308; Del Corno u. a. (1997) 304; Chrétien (1985) 67 f.; Hernández de la Fuente (2008) 80. 
Magus, dem Gegenspieler des Petrus, die Lesung eines Buches eingeflochten ist, welches Appion in einer einzigen Nacht schreibt, um es dann Klemens zu übergeben:

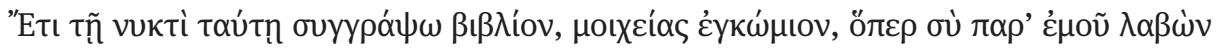

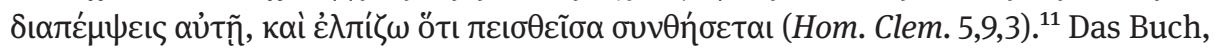
das Klemens den Anwesenden sodann vorliest, ist eine Zusammenstellung von mythischen Beispielen von Treuelosigkeit und Ehebruch, die einen umfangreichen Katalog der wichtigsten heidnischen Götter, darunter auch Dionysos, und ihrer männlichen Geliebten enthält (Hom. Clem. 5,15,1-3): ${ }^{12}$

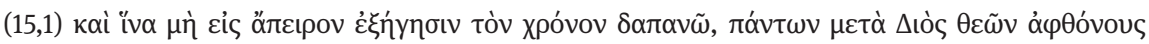

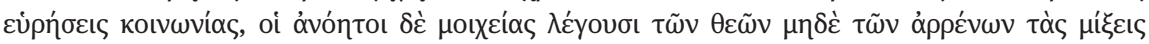

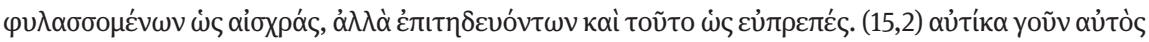

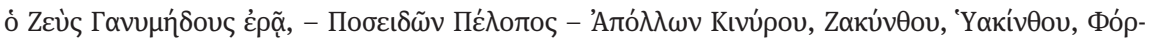

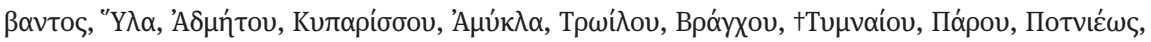

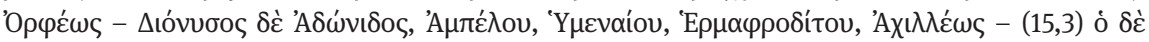

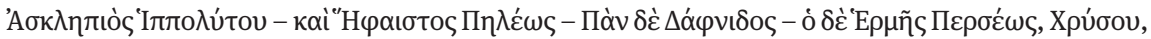

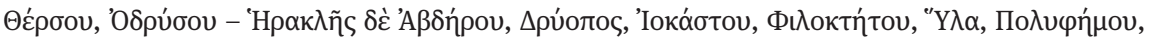

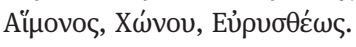

(15,1) Doch damit ich die Zeit nicht für einen endlosen Bericht verschwende: Du wirst zahllose sexuelle Vereinigungen aller Götter nach Zeus entdecken. Die Unverständigen nannten das Ehebruch, während sich die Götter nicht einmal vor Geschlechtsverkehr mit Männern als etwas Schändlichem hüteten; sie gaben sich vielmehr auch dem als etwas Anständigem hin. (15,2) Zum Beispiel war Zeus selbst in Ganymedes verliebt, Poseidon in Pelops, Apollon in Kinyras, Zakynthos, Hyakinthos, Phorbas, Hylas, Admetos, Kyparissos, Amyklas, Troilos, Branchos, Tymnaios, Paros, Potnieus und Orpheus, Dionysos aber in Adonis, Ampelos, Hymenaios, Hermaphroditos und Achilleus. (15,3) Asklepios aber in Hippolytos, und Hephaistos in Peleus, Pan aber in Daphnis, Hermes aber in Perseus, Chrysas, Thersos und Odrysos, Herakles aber in Abderos, Dryops, Iokastos, Philoktetes, Hylas, Polyphemos, Haimon, Chonos und Eurystheus.

Ampelos erscheint wie selbstverständlich in die Liste der Göttergeliebten aufgenommen. Trotz der spärlichen Zeugnisse lässt die Erwähnung in den Pseudo-Klementinen dennoch eine gewisse mythologische Tradition vermuten, von welcher sich Nonnos zur Gestaltung seines Ampelos inspirieren ließ. Im selben Atemzug mit Ampelos wird auch ein weiterer Geliebter des Dionysos genannt, Hymenaios, der ebenfalls in den Dionysiaka in Erscheinung tritt und in enger Verbindung zur Ampelos-Figur steht. ${ }^{13} \mathrm{Nicht}$ ausgeschlossen werden kann, dass der Dichter sich zur Modellierung dieser beiden Figuren Handbücher oder Namenslisten bediente, die jedoch für die moderne Forschung nicht mehr greifbar sind. ${ }^{14}$

11 Text: Rehm u. Strecker (1992) 95.

12 Text: Rehm u. Strecker (1992) 98f.; Übersetzung: Wehnert (2010) 120.

$13 \mathrm{Zu}$ Ampelos und Hymenaios vgl. S. 56f. und 234-237.

14 Vgl. Agosti (2004) 290. - Ob sich von dem auf kleinasiatischen Votivtäfelchen belegten Zeus Ampeleites eine Verbindung zu Ampelos bei Nonnos herstellen lässt, ist fraglich; zu Zeus Ampeleites vgl. Robert u. Robert (1974) 293; Robert (1983); Bowersock (1990) 44f. - Robert (1983) 541 f. sieht in Zeus Ampeleites keinen Schutzgott des Weinbaus, sondern der Pferde und Rinder, da er auf den Tafeln 
Größere Chancen auf eine Identifikation des Ampelos-Mythos als die Literatur versprechen bildliche Darstellungen. Einige Zeugnisse aus der Skulpturen- und Reliefkunst werden sowohl in der Sekundärliteratur zu den Dionysiaka als auch in archäologischen Arbeiten als Repräsentationen des Ampelos gedeutet. Die folgende Spurensuche setzt sich nicht eine vollständige und systematische Aufarbeitung der Ampelos-Darstellungen oder etwa eines bestimmten ikonographischen Typs der SatyrDarstellung zum Ziel, sondern will eine kritische Revision der Forschungsmeinungen bieten und die Frage stellen, ob die Interpretation von Satyr-Figuren als Ampeloi jeweils haltbar ist.

\title{
3.2 Ampelos in der bildenden Kunst
}

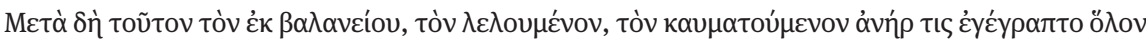

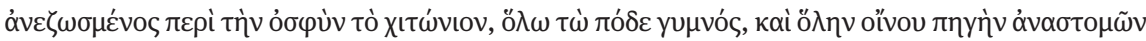

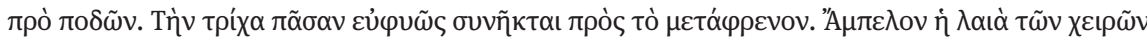

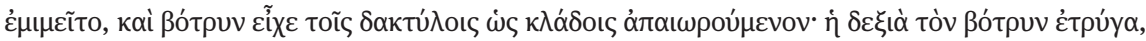

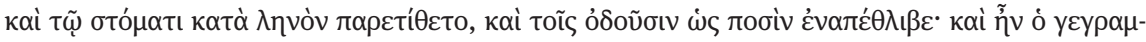

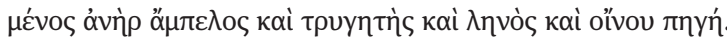

Nach diesem, dem Mann, der aus dem Baderaum getreten war, der gebadet hatte, der unter der Hitze stöhnte, war ferner ein Mann gemalt, der sich das ganze Gewand um die Lenden geschlungen hatte, an den Beinen von oben bis unten nackt war und vor seinen Füßen eine richtiggehende Weinquelle erschloß; die Haare hatte er sich auf den Rücken alle sehr hübsch zusammengebunden. Seine linke Hand ahmte einen Weinstock nach und hielt eine Traube, die an den Fingern wie an Zweigen hing; seine Rechte las die Traube ab und versorgte damit den Mund wie eine Kelter, und mit den Zähnen zerdrückte er sie wie mit Füßen, und so war der dargestellte Mann Weinstock, Leser, Kelter und Weinquelle zugleich. ${ }^{15}$
\end{abstract}

Dieser Auszug aus dem byzantinischen Roman Hysmine und Hysminias $(4,11)$ des in die zweite Hälfte des 12. Jhs. zu datierenden Eustathios (oder Eumathios) Makrembolites zeigt die Ekphrasis des Monatsbildes für September, eines Gemäldes, das an der Mauer des Gartens des Sostratos angebracht ist und das der Protagonist Hysminias bestaunt. ${ }^{16}$ Dargestellt ist eine männliche Person beim Weinkeltern, wobei diese gleichsam eine vierfache Metamorphose vollzieht, welche Ampelos' Schicksal bei Nonnos in Erinnerung ruft.

stets zusammen mit Gruppen dieser Tiere dargestellt ist. Dies könnte die herausragende Rolle des Stieres in der Ampelos-Episode erklären: Der Satyr begegnet einem Stier, führt eine rituelle Schmückung des Tieres durch und kommt durch selbiges schließlich zu Tode; Nonnos könnte den agrarischen Aspekt des Kultes für Zeus Ampeleites aufgegriffen und in seinem Mythos über Ampelos verarbeitet haben; zur Funktion von Tieren in den Dionysiaka vgl. Miguélez Cavero (2014a).

15 Text: Marcovich (2001) 40 f., Übersetzung: Plepelits (1989) 108; vgl. auch Eustathios Makrembolites, Hysmine und Hysminias 4,18,8.

16 Zum Roman des Eustathios Makrembolites siehe Plepelits (1989) 1-81. 
Ähnliche Darstellungen, wie in der Ekphrasis des Eustathios beschrieben, finden sich auch in der bildenden Kunst der Spätantike, die in der Nonnos-Forschung vielfach unreflektiert als Ampelos-Illustrationen gedeutet werden. Darstellungen des Ampelos wurden immer wieder für unterschiedliche Gattungen und Medien der bildenden Kunst konstatiert, so etwa für einige Beispiele aus der Skulpturen- und Reliefkunst. Dazu zählt ein männlicher Torso mit Weinranken aus der ersten Hälfte des 2. Jhs. n. Chr., der heute im Moskauer Puschkin-Museum verwahrt und bald mit Dionysos, bald mit Ampelos identifiziert wird. ${ }^{17}$ Ein Terrakottafragment von ähnlicher ikonographischer Gestaltung ist für Nonnos' Ampelos von besonderem Interesse: Das im Musée du Louvre aufbewahrte, aus Oberägypten stammende und ins 4. Jh. n. Chr. datierte Stück ist in unmittelbare örtliche und zeitliche Nähe zu Nonnos zu rücken; es zeigt ebenfalls eine männliche Figur mit Weintrauben und wird von Pierre du Bourguet in dionysischen Kontext gestellt und von Daria Gigli Piccardi als Ampelos identifiziert. ${ }^{18}$ Nach du Bourguet spricht der ikonographische Typus der Metamorphose, der auch in einem Hochrelief mit der sich in den Lorbeer verwandelnden Daphne vorliege, für eine Darstellung des Satyrn. ${ }^{19}$ Doch ist bei dieser Deutung Vorsicht geboten, da sich vergleichbare Darstellungen finden, die zweifelsfrei Dionysos, den Weingott selbst, zeigen: so etwa auf einer Wandmalerei in der Casa del Centenario in Pompeji, auf der Dionysos gänzlich von Weintrauben umhüllt gezeigt wird, ${ }^{20}$ oder in einer Bronzefigurine aus München, die den Weingott von einem Weinstock umrahmt wiedergibt. ${ }^{21}$

Als Ampeloi werden auch drei männliche Figuren identifiziert, die, so die Deutungsansätze, eine Metamorphose in den Weinstock vollziehen: Aus dem British Museum stammt ein von H. B. Walters beschriebenes Terrakotta-Fragment, das um die Zeitenwende entstand und drei Satyrn zeigt. Die beiden äußeren Figuren musizieren der linke Satyr hält ein Tympanon in Händen, der rechte Zimbeln - und umgeben so die mittlere Figur, deren Unterleib in Weinranken und Trauben übergeht und folglich als Ampelos gedeutet wird..$^{22}$ Außerdem deutet Robert Turcan die Figur am linken Rand eines spätantiken Sarkophags aus Acqua Traversa bei Gallicano, der bereits von Salvatore Aurigemma beschrieben und abgebildet wird und die Darstellung eines dionysischen Zuges bietet, als den sich in einen Weinstock verwandelnden Ampelos; ${ }^{23}$

17 Vgl. Britova (1960).

18 Vgl. du Bourguet (1967) 36f.; Gigli Piccardi (2003) 630f. Anm. 31.

19 Vgl. du Bourguet (1967) Abb. 13 im Anhang; vgl. Thomas (2000) Abb. 52; Gigli Piccardi (2003) 631 Anm. 31; Hernández de la Fuente (2008) 283.

20 Vgl. Mau (1928) 40; Schefold (1957) 273; Hamdorf (1986) 98.

21 München, Archäologische Staatssammlung München Inv.-Nr. 3038, vgl. Manfrini-Aragno (1987) Abb. 67. - Figuren mit ähnlicher Ikonographie, die jeweils nicht als Ampelos, sondern als Dionysos gedeutet werden, sind in Ägypten mehrfach belegt, vgl. Thomas (2000) Abb. 37, 39, 40 und 80.

22 Vgl. Walters (1903) 387 D 534; Chrétien (1985) 69 Anm. 1; zur Abbildung siehe auch Kerényi (1994) Abb. 67, der die Darstellung als „Epiphanie des göttlichen Kindes aus dem Weinstock“ deutet. 23 Vgl. Aurigemma (1954) 17 f. und Taf. VII; Turcan (1961) 162-166, Abb. 5; Turcan (1966) 341343, Taf. 61a-b und 62b-c; LIMC III/2, 435 Abb. 94. 
dieser Interpretation schließt sich auch Francis Vian an. ${ }^{24}$ Herangezogen wird auch eine Statuengruppe aus der zweiten Hälfte des 2. Jhs. n. Chr., die ebenfalls im British Museum aufbewahrt und zumeist als Dionysos-Ampelos-Gruppe gedeutet wird..$^{25}$ Die allmählich in einen Weinstock übergehende Figur ist mit Weintrauben behängt und dient dem größeren Dionysos als Stütze.

Auch dieser Interpretation ist jedoch nur mit einigem Vorbehalt zuzustimmen, da sich die Figurengruppe Dionysos mit Satyr mehrfach in der antiken Kunst findet, wobei nirgendwo, etwa durch eine Beischrift, eine unzweifelhafte Identifizierung mit Ampelos möglich ist. Die zahlreichen Beispiele für diese Gruppe, etwa aus der Skulpturenund Reliefkunst, lassen vermuten, dass es sich um einen ikonographischen Typus handelt. ${ }^{26}$ In den meisten Fällen wird Dionysos, der durch die Attribute Weintraube, Weinlaubkranz und Trinkgefäß als solcher kenntlich ist, dargestellt, wie er sich auf seinen kleineren, jugendlichen Begleiter, „un jeune Satyre“ ${ }^{\text {27 }}$, stützt. Letzterer wird meist ebenfalls durch Weintrauben sowie durch einen Fellumhang und einen Hirtenstab (pedum) bezeichnet. In Varianten dieser Figurengruppe nimmt gelegentlich ein Silen oder Pan den Platz des jungen Satyrn an der Seite des Dionysos ein. ${ }^{28}$ Die Deutung der Figuren als Dionysos und Ampelos findet sich dennoch nicht nur in der Literatur zu den Dionysiaka des Nonnos, sondern gelegentlich auch in archäologischen Arbeiten, so etwa bei der dionysischen Figurengruppe auf einem marmornen Puteal aus Madrid. ${ }^{29}$

Zahlreiche Beispiele für ebendiese Figurengruppe finden sich auch in der spätantik-kaiserzeitlichen Mosaikkunst. Die Beispiele für Dionysos und seinen jungen Begleiter mit Fellbekleidung, Weinranken und pedum begegnen sowohl im östlichen

24 Vgl.Vian (1995) 70 Anm. 2 und 74 f. Anm. 2; Matz (1969) 327 f. ad Taf. 202 Abb. 178 bezeichnet die Figur lediglich als Satyr, nicht als Ampelos. - Ein fragliches Zeugnis wird in der französischen BudéEdition der Dionysiaka verzeichnet: Einer Hypothese zufolge könnte es sich bei einer fragmentarisch erhaltenen Darstellung eines Stieres, der unter einem Exedra-Mosaik in der Villa Erculia in Piazza Armerina zu erkennen sei, um eine Szene mit Ampelos auf dem Stier handeln; die umgebenden Darstellungen (Lykurg und Ambrosia) gehören ebenfalls der dionysischen Sphäre an; vgl. Gentili (1959) Abb. 12; Settis (1975) 969; Dunbabin (1999) 137 Abb. 139; Chrétien (1985) 69 Anm. 2 von Pierre Chuvin; Gigli Piccardi (2003) 631 Anm. 31. - Ebenso unklar ist die Identität einer Statuette im Roemer- und Pelizaeus-Museum in Hildesheim; vgl. LIMC I/1, 689f.; Hernández de la Fuente (2007) 197 Anm. 48.

25 Siehe Roscher I/1, 292; Combe (1818) Taf. XI; Müller K. O. (1848) 598; Koehler (1853) 25; Ménard (1878) 585; Reinach (1897) 387; Turcan (1966) 342; LIMC I/1, 690; Sittl (1985) 821, 831, 845. Anderer Auffassung ist lediglich Smith A. H. (1904) 50f. Nr. 1636, der in der kleineren Figur nicht Ampelos, sondern eine weibliche Figur, vielleicht Ambrosia, sehen will; vgl. dazu auch Klein (1921) 81. 26 Zu diesem Typus siehe auch Dunbabin (1978) Taf. LXX,178; Taf. LXXI,182; Manfrini-Aragno (1987) 91f., sowie Abb. 125 - 127; Pochmarski (1990) passim sowie Taf. 58 Abb. 2, Taf. 63 Abb. 2, Taf. 67 Abb. 1, Taf. 69 Abb. 2, Taf. 74 Abb. 2, Taf. 76 Abb. 1, Taf. 77 Abb. 1, Taf. 78 Abb. 2, Taf. 79 Abb. 2; Hackländer (1996) 230 Abb. 4.

27 Manfrini-Aragno (1987) 91.

28 Siehe etwa Manfrini-Aragno (1987) Abb. 128-132.

29 Siehe Hübner (1862) 144-146; García y Bellido (1951). 
als auch im westlichen Teil des Imperiums, etwa in Antiochia/Antakya, Burgos, Complutum/Alcalá de Henares und Köln. ${ }^{30}$ Ein Blick auf die beiden spanischen Mosaiken aus Burgos und Alcalá de Henares soll stellvertretend auch für die übrigen Beispiele stehen: Das wohl in die zweite Hälfte des 4. Jhs. n. Chr. zu datierende Mosaik aus einer römischen Villa in Valdearados (Burgos) zeigt in zwei übereinander angeordneten Registern den Triumph des Dionysos. ${ }^{31}$ Im unteren Register wird der triumphierende Dionysos auf einem von zwei Panthern gezogenen Wagen und in Begleitung von zwei Figuren gezeigt, im oberen steht der Gott, der von insgesamt elf Figuren, darunter auch einem jungen Satyrn, flankiert wird. Das Mosaik aus einer römischen Villa bei Alcalá de Henares bietet denselben ikonographischen Typus: Der Weingott, dessen Haupt mit einem Weinlaubkranz und mit Trauben geschmückt und der mit einem Umhang bekleidet ist, wird von einem Alten und einem Jungen begleitet. ${ }^{32}$ Um eine Variation zu diesem Typus handelt es sich bei Satyrn, die losgelöst von Dionysos, jedoch in dionysischem Gefolge anzutreffen sind, dies oftmals im Kontext des Triumphzuges über die Inder oder Dionysos' Begegnung mit Ariadne, so etwa auf Mosaikbildern aus Antiochia/Antakya, ${ }^{33}$ Nea Paphos auf Zypern, ${ }^{34}$ Thysdrus/ El Jem in Tunesien ${ }^{35}$ oder dem spanischen Mérida. ${ }^{36}$

Die Häufigkeit dieser beiden ikonographischen Typen, des Dionysos mit Stützfigur und des durch charakteristische Attribute kenntlich gemachten Satyrn, und deren Verbreitung in der gesamten Mittelmeerwelt stehen in scharfem Kontrast $\mathrm{zu}$ den spärlichen literarischen Zeugnissen für Ampelos. Die Deutung des Satyrn in Gemeinschaft des Dionysos als Ampelos erscheint somit vor diesem Hintergrund problematisch. Die zahllosen spätantiken Darstellungen des Dionysos und seines Thiasos lassen es wenig glaubhaft erscheinen, dass es sich bei dem Satyrn an Dionysos' Seite tatsächlich immer um Ampelos handelt. Das zeigt, dass Spuren des Ampelos-Mythos auch in der bildenden Kunst kaum in Erscheinung treten.

30 Zu einigen der Abbildungen vgl. auch Pochmarski (1990) Taf. 29 Abb. 1 und 2; zu den Mosaiken von Antiochia mit den genannten Satyr-Darstellungen vgl. LIMC III/2, 413 Abb. 81 und 82 sowie Cimok (2000) 51 und 59; zu Alcalá de Henares-Complutum siehe Fernández-Galiano (1984a) 148-186; Guardia (1989); Blázquez (1993) 184-187; Parrish (2004) 75 f.; zum Kölner Mosaik siehe Doppelfeld (1967) Abb. 17.

31 Vgl. Argente Oliver (1979) 45-58, Taf. II-VI; Blázquez (1993) 191f., 307-317, 321-326; Pochmarski (1990) 92 f.; López Monteagudo (1999); Parrish (2004) 79. - Das Nebeneinander zweier unterschiedlicher Szenen zum selben narrativen Themenkomplex ist auch für andere Mosaiken belegt und stellt ein charakteristisches Stilmerkmal der spätantiken Kunst dar; vgl. López Monteagudo (1999) $35 \mathrm{f}$.

32 Siehe López Monteagudo (1999) 42.

33 Siehe die Abb. in: Cimok (2000) 161.

34 Siehe die Abb. in: Kondoleon (1995) 194 Abb. 120.

35 Siehe die Abb. in: Parrish (1984) Taf. 39d.

36 Siehe Dunbabin (1978) Taf. LXIX Abb. 175, außerdem Taf. LXX Abb. 178 und 179, Taf. LXXI Abb. 182; Parrish (2004) $80 \mathrm{f}$. 
Eine Wiederaufnahme des antiken Ampelos-Mythos durch bildende Künstler der Neuzeit ist lediglich vereinzelt $\mathrm{zu}$ bemerken. So findet sich etwa in Alexander Hendersons The History of Ancient and Modern Wines als Illustration einer Initiale der Weingott sitzend und ihm zur Seite ein junger Satyr, der in der Abbildungsbeischrift mit „Ampelus“ bezeichnet ist. ${ }^{37}$ Im Unterschied zu den antiken Beispielen mit Dionysos, der sich auf einen anonymen Satyrn stützt, wird der Weingott hier bärtig, sitzend und gänzlich bekleidet gezeigt, Ampelos hingegen als nackter Jüngling, eine Illustration, die sich auch im zweiten Band der Antiquités étrusques, grecques et romaines von François-Anne David und Pierre d'Hancarville findet. ${ }^{38}$ In keinem der beiden Fälle finden sich nähere Angaben zur Provenienz der Darstellung; David und d'Hancarville schreiben den zugrunde liegenden Mythos „Nonnius“ (sic) zu, was ihre Kenntnis der Dionysiaka impliziert.

Eine zweifelsfreie Rezeption der nonnianischen Ampelos-Episode liegt im Fall des Hymnus oft Lof-Sanck van Bacchus, waer in't ghebruyck ende misbruyck vande wijn beschreven wort vor, den Daniel Heinsius in seinen Band Nederduytsche Poemata (Amsterdam 1618) aufnimmt, in dem er auch das Schicksal des Ampelos nach dem Vorbild des Nonnos erzählt (Abb. 1). ${ }^{39}$ Eine zugehörige Abbildung in zwei Szenen veranschaulicht die Geschehnisse: links im Hintergrund die Tötung des Ampelos durch den Stier, bei der auch Dionysos anwesend ist; rechts die Verwandlung des Ampelos in einen Weinstock, den der anwesende Gott sogleich betrachtet. Dasselbe ikonographische Schema findet sich in einem weiteren Stich aus demselben zeitlichen und geographischen Umfeld (Abb. 2). Das Bildmotiv ist, abgesehen von der reduzierten Wiedergabe der Vegetation, beinahe ohne Änderung übernommen: Tod und Auferstehung des Ampelos im Beisein des Dionysos werden auch hier in zwei Szenen umgesetzt. ${ }^{40}$

Heinsius’ Beschäftigung mit den Dionysos-Mythen und seine Kenntnis der Dionysiaka steht in enger Verbindung zum gesteigerten Interesse am Autor seit der Erstedition durch Gerart Falkenburg 1569. Wenige Jahre vor der dichterischen Bearbeitung der Dionysiaka durch Heinsius, nämlich 1605, hatte der Rostocker Gelehrte Eilhard Lubin die Dionysiaka ins Lateinische übersetzt, ${ }^{41} 1625$ folgte die erste Übersetzung in

37 Siehe Henderson (1824) XV und 37.

38 Siehe David u. d'Hancarville (1787) 109 und Taf. 5.

39 Heinsius (1618) 111. - Der Originalstich von 1616, ausgeführt von Jacob Matham, David Vinckboons und Willem Janszoon Blaeu, befindet sich unter der Objektnummer RP-P-OB-102.855 im Rijksmuseum Amsterdam. - In die von Martin Opitz angefertigte und 1622 publizierte Übersetzung von Heinsius' Werk unter dem Titel Hymnus oder Lobgesang Bacchi, darinnen der gebrauch und missbrauch des Weins beschrieben wird fand die Illustration keinen Eingang. - Heinsius setzt sich auch an anderer Stelle mit Nonnos auseinander: In seiner Dissertatio zu Nonnos legt er Zeugnis ab für seine frühe und zunächst begeisterte Beschäftigung mit dem Autor, vgl. Gärtner U. (2008).

40 Die Originalillustration von Pieter Serwouters und Jacob Matham, datiert zwischen 1616 und 1657, befindet sich ebenfalls im Rijksmuseum Amsterdam (Objektnummer: RP-P-1886-A-10528).

41 Dieselbe lateinische Übersetzung findet sich in der nur ein Jahr später (1606) erschienenen Zusammenstellung der griechischen Profandichtung der Antike durch den Schweizer Juristen und Ge- 
eine moderne Fremdsprache, und zwar ins Französische durch Claude Boitet de Frauville. Die Barockzeit hatte aufgrund seines Stils eine besondere Affinität zu Nonnos und seiner Poetik, wofür auch die Dichtungen Giovan Battista Marinos Zeugnis ablegen. ${ }^{42}$ Es darf also nicht verwundern, dass gerade aus dieser Epoche die einzigen zweifelsfreien Illustrationen von Szenen der nonnianischen Ampelos-Episode stammen. Das besondere Interesse des Barock an den Dichtungen des Nonnos konzentriert sich hier auf die flämisch-niederländische Philologie und ihr künstlerisches Umfeld.

Eine systematische Untersuchung der Ampelos-Rezeption in dieser Zeit würde den Rahmen der vorliegenden Arbeit sprengen und muss daher künftigen Forschungen vorbehalten bleiben. Es soll lediglich Folgendes festgehalten werden: Während sich vereinzelt eine echte Ampelos-Rezeption in Literatur und Kunst ab dem 17. Jh. nachweisen lässt, bleibt die Figur in der Antike, mit Ausnahme von Ovid, den PseudoKlementinen und den Dionysiaka, im Dunkeln. Dem weitgehenden Fehlen jeglicher literarischer Belege ist eine Vielzahl an bildlichen Darstellungen hinzuzurechnen, die allesamt keine eindeutigen Indizien für eine Identifikation mit Ampelos liefern. Die knappe Notiz zu Ampelos bei Ovid und die wie selbstverständliche Reihung des Ampelos unter die Geliebten des Weingottes in den Pseudo-Klementinen lassen lediglich die Vermutung zu, dass Ampelos als Figur des Mythos bereits ab dem Hellenismus bekannt war. Nonnos knüpft mit seinem Mythos offenbar an eine bestehende Tradition an, die jedoch aufgrund des Quellenmangels in ihren Einzelheiten nicht mehr greifbar ist. Auffällig ist das ungleiche Verhältnis der äußerst ausführlichen Behandlung des Mythos um Ampelos in den Dionysiaka und die Kargheit der sonstigen literarischen Quellen. Während Ovid die Episode um Dionysos und seinen Lieblingssatyrn in lediglich zwölf Versen und überdies in einer Alternativversion abhandelt, komponiert Nonnos zweieinhalb Bücher. Es stellt sich nun die Frage, aus welchen Elementen Nonnos seine sonst unbekannte Geschichte zusammensetzt. Schöpft er vielleicht doch aus literarischen Quellen, um seinen Ampelos $\mathrm{zu}$ formen, und wenn nicht aus einem eigenen Ampelos-Mythos, so womöglich aus anderen, vergleichbaren Mythen? Um mögliche Antworten darauf zu finden, wird im Folgenden die AmpelosFigur, so wie sie Nonnos in seiner Episode konzipiert, vorgestellt.

\subsection{Ampelos im Porträt}

Mit dem sprechenden Namen Ampelos, „der Weinstock“, bietet Nonnos eine Lesehilfe und zugleich einen wesentlichen Anhaltspunkt für das kompositorische Ziel der Bücher 10-12. Der aus der mythischen Tradition nahezu unbekannte Satyr und junge

lehrten Jacques Lect; für genaue bibliographische Angaben siehe Lectius (1606) im Literaturverzeichnis.

42 Vgl. Gonnelli (2003) 26-31. 
Geliebte des Dionysos erhält bei seinem ersten Auftritt im 10. Buch der Dionysiaka vom Dichter ein ausführliches Porträt zuerkannt, das unmittelbar an die Badeszene mit Dionysos und den Satyrn anschließt. Aus dem Kollektiv der Satyrn wird der bedeutendste Vertreter herausgegriffen, der zusammen mit Dionysos den Handlungskern der folgenden eineinhalb Bücher bildet und darüber hinaus für die Etablierung des Dionysos als Weingott von immenser Wichtigkeit ist. Thematisiert wird zunächst Ampelos’ Aussehen (Nonn. D. 10,175-192): ${ }^{43}$

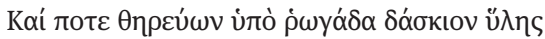

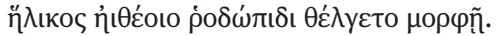

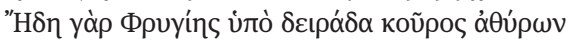

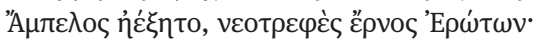

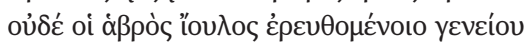

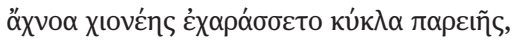

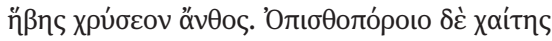

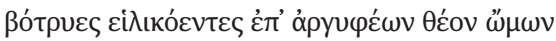

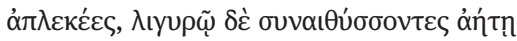

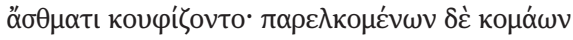

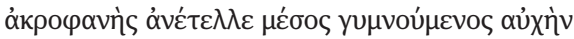

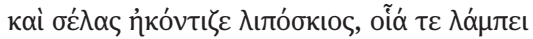

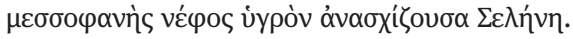

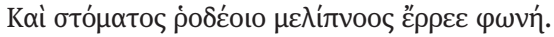

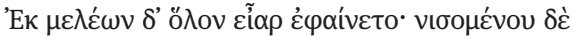

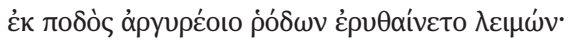

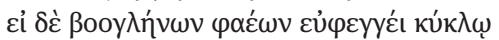

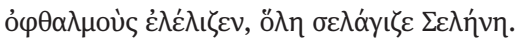

(175) Und als er einmal im felsigen Dickicht des Waldes auf der Jagd war, erfreute er sich an der rosengesichtigen Gestalt eines gottgleichen Altersgenossen. Denn schon war beim Spielen unten an einem Gebirgskamm in Phrygien ein Jüngling herangewachsen, Ampelos, ein jüngst großgezogener Spross der Eroten. Und er hatte noch keinen schönen Bartflaum am rosigen Kinn, (180) und bartlos war noch das Rund der schneeweißen Wange gezeichnet, die goldene Blüte der Jugend. Und die spiralförmigen Trauben seiner nach hinten fallenden Haarmähne liefen auf die silbern glänzenden Schultern, ungeflochten, und durch einen heftigen Windstoß schlugen sie zusammen und wurden durch einen Luftzug emporgeweht. Und wenn er die Haare zur Seite zog, (185) wurde der entblößte Hals oben in der Mitte sichtbar und warf einen schattenlosen Glanz, wie wenn Selene mitten in einer feuchten Wolke erscheint und sie aufreißt. Und aus dem rosigen Mund floss eine Stimme von süßem Hauch. Aus den Gliedern strahlte ganz und gar der Frühling hervor. Wenn er dahinschritt, (190) färbte sich aus seinem silbrigen Fuß die Wiese rot von Rosen. Und wenn er im strahlenden Rund seine Augen mit Pupillen glänzend wie bei einer Kuh bewegte, dann leuchtete der Vollmond.

Zunächst fällt der Blick auf den bevorzugten Aufenthaltsbereich des Jünglings, den dichten Wald der phrygischen Berge (Nonn. D. 10,175-177), sodann wird in aller Ausführlichkeit durch eine Reihe von Wendungen sein jugendliches Alter betont:

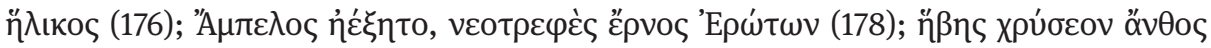


$(10,181)$. Auf die Bartlosigkeit und das Farbenspiel vom Rot und Weiß des Gesichts (Nonn. D. 10,179f.) folgen die vom Wind bewegten Haarlocken (181-187), Mund und Stimme (188) sowie die Gliedmaßen (189), Füße (189f.) und Augen (191f.). Die Abfolge der Beschreibung vom Kopf bis zu den Füßen ist in von der Rhetorik beeinflussten Personenbeschreibungen geläufig. ${ }^{44}$ Mit den Schönheitstopoi schöpft der Dichter nicht zum ersten Mal in den Dionysiaka aus der rhetorischen Tradition: Bereits Kadmos wird bei seiner Ankunft auf Samothrake in ähnlicher Weise beschrieben (Nonn. D. 4,126142) ebenso wie Semele, die Mutter des Dionysos (7,222-224, 236-252) und einige weitere Figuren in den Dionysiaka. ${ }^{45}$ Für einzelne Motive, die in der Ampelos-Ekphrasis bedient werden, lassen sich auch Beispiele in der griechischen Dichtung vor Nonnos finden: Der Vergleich der Schönheit mit dem Mond ist etwa bei Hesiod fr. 252 (Merkelbach u. West) und Sappho fr. 96,6 -9 (Lobel u. Page) sowie bei Theokrit (Theoc. 2,79) und Ovid (Ov. met. 4,329-333) anzutreffen. ${ }^{46}$ Ähnlich wie bei Ampelos fallen auch in einer Ode Pindars Iasons Locken auf dessen Rücken (Pi. P. 4,82f.), ${ }^{47}$ und der Topos des rosenfarbenen Mundes taucht in der Romanliteratur des Longos und Achilleus Tatios wieder auf (Longus 1,18,1; Ach. Tat. 1,4,3; 2,1,3). ${ }^{48}$ Nonnos knüpft bei der Gestaltung seiner Figur an überkommene narrative Techniken an, die den Satyrn zunächst nicht mehr als ein Produkt literarischer Traditionen erscheinen lassen. ${ }^{49}$

Trotz der schematischen Elemente und der feststehenden Topoi gelingt es Nonnos dennoch, dem Porträt eine individuelle Note zu verleihen. Mit der Ekphrasis des Ampelos verfolgt er ein bestimmtes narratives Ziel: die Vorbereitung auf Dionysos' Hauptattribut, den Wein. ${ }^{50}$ Im Verlauf der Ekphrasis erhält der Satyr mehrfach Charakteristika übertragen, die seiner späteren Erscheinungsform nahekommen, etwa das Motiv der wachsenden und blühenden Vegetation, das bereits bei der ersten na-

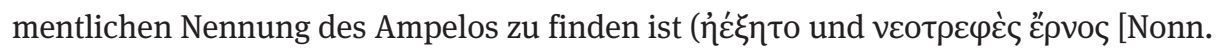

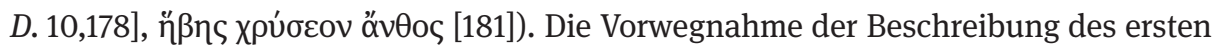
Weinstocks kumuliert in der Metaphorik der Sprache in Vers 182, wo Ampelos' Locken

44 Vgl. Gigli Piccardi (2003) $700 f$.

45 Insgesamt finden sich zehn ausführlichere Ekphrasen auf Personen: außer für (1.) Ampelos, (2.) Kadmos und (3.) Semele auch für (4.) Kalamos (Nonn. D. 11,370 - 384), (5.) Hymenaios (13,84 f., 90 92; 29,15-178), (6.) Hymnos (15,204-206, 210), (7.) Nikaia (15,220-243), (8.) Chalkomede (34,106-121), (9.) Beroe (41,250 - 262) und (10.) Pallene (48,116-123); vgl. Chrétien (1985) 142 Anm. ad 175 - 192 mit weiteren Literaturangaben. - Auch der in der Tradition nonnianischer Dichtung stehende Musaios bedient sich dieser Technik, vgl. Kost (1971) 223 - 272 zu den Versen Musae. 55 - 66; zum Schönheits-Topos in den Dionysiaka vgl. auch D’Ippolito (2013) $284 \mathrm{f}$.

46 Vgl. Del Corno u. a. (1997) 304; Chrétien (1985) 143f. Anm. ad 187.

47 Vgl. Chrétien (1985) 143 Anm. ad 184.

48 Vgl. Chrétien (1985) 144 Anm. ad 188. - Vgl. auch die Schönheit des Dionysos in E. Ba. 235 f., 455 457. Ähnliche Schönheitstopoi finden sich auch im byzantinischen Roman, vgl. etwa die Ekphrasis zweier Mädchen im mittelalterlichen Versroman Floros und Plaziaflore 805 - 811, Cupane (1995) 508f. 49 Vgl. auch in der christlichen Literatur Prud. perist. 13,28-32, wo der Märtyrer Cyprian von Karthago vor seiner Konversion als bartloser Jugendlicher mit langem Haar und hübschem Gesicht dargestellt wird und erst danach das Aussehen eines Erwachsenen erhält, siehe Malamud (1989) 122. 50 Vgl. Del Corno u. a. (1997) 304; Gigli Piccardi (2003) 700f. Anm. ad 175-192. 
als ßótрuєৎ, „Trauben“, bezeichnet werden. Für die Angleichung von Haar und

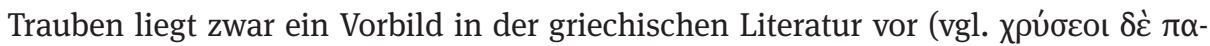

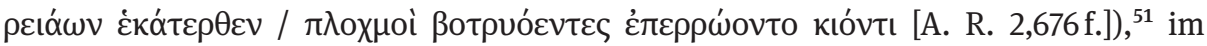
Unterschied dazu baut Nonnos jedoch ein einzelnes Motiv zu einer ganzen Episode aus und arbeitet kontinuierlich an seinem poetischen Plan, indem mehrfach auf die rote

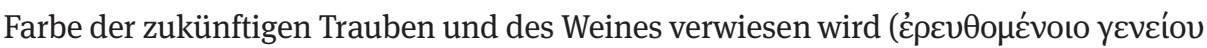

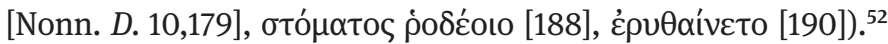

Der Verweis auf den Weingott erfolgt nicht nur durch das Ampelos-Porträt, sondern auch durch die übrigen Personenbeschreibungen. Insbesondere diejenigen Figuren erhalten eine besonders ausführliche Charakterisierung, die mit Dionysos und dem dionysischen Wesen in enger Verbindung stehen: Kadmos als der Prototyp des kommenden Dionysos, Semele als Mutter ihres göttlichen Sohnes sowie Kalamos, Hymenaios, Hymnos, Nikaia, Chalkomede, Beroe und Pallene, die gleichfalls der dionysischen Sphäre zuzurechnen sind. Die Auswahl der Figuren, denen der Erzähler eine Ekphrasis zugesteht, ist also keineswegs zufällig, sondern erfolgt gezielt durch die unmittelbare Bindung an die Dionysos-Handlung sowie durch Verweise und motivische Entsprechungen auf ebendiese.

Die Kompositionslinie, die auf Höhepunkt und Ende der Episode hin ausgerichtet ist, wird in der folgenden Rede des Dionysos an Zeus weitergeführt (Nonn. D. 10,193216) und kumuliert in einem Enkomion auf Ampelos und den Weinstock (12,207289)..$^{53}$ In der Rede an Zeus setzt der Dichter das Ampelos-Porträt insofern fort, als er den Sprecher Dionysos Fragen zur (göttlichen) Herkunft des Satyrn stellen lässt. ${ }^{54}$ Dionysos bringt im Verlauf der Rede auch seine eigene Genealogie zur Sprache, indem er auf seine Zugehörigkeit zum Geschlecht der Olympier und auf Ampelos' Unsterblichkeit verweist. Dieselben Motive werden in der Rede des Dionysos am Ende des 12. Buches wieder aufgenommen und ausgebaut. In einer Art Verteidigungsrede in eigener Sache, in der Dionysos seine Göttlichkeit durch rhetorische Argumentation zu belegen sucht, bildet der bereits in den Weinstock verwandelte Ampelos den Ausgangspunkt.

51 Vgl. Chrétien (1985) 143 Anm. ad 182.

52 Zum Bereich der Vegetation ist auch die Mond-Metaphorik in Nonn. D. 10,185-192 zu zählen, wonach bereits nach antiker Vorstellung der Mond für das Wachstum der Pflanzen verantwortlich ist, vgl. Gigli Piccardi (2003) 702. - Zum Bild der Selene in diesen Versen siehe auch S. 69f. - Mit der

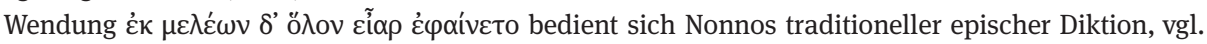

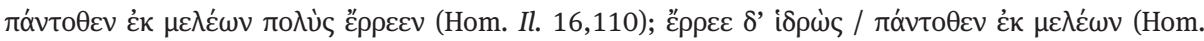

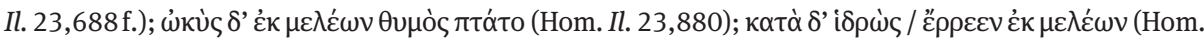
Od. 11,599f.; Q. S. 2,531 und 557; 4,159; 5,37; 8,203 und 313). Der Dichter der Dionysiaka verleiht dem epischen Wortmaterial eine zusätzliche erotische Note, vgl. [D.] Or. 61,10 mit einer Beschreibung

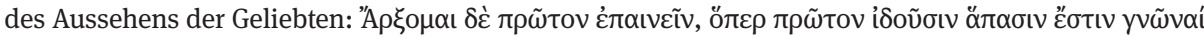

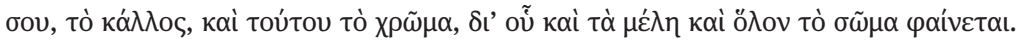

53 Zur Rede an Zeus siehe auch Kröll (2014).

54 Zur Verbindung des Schönheits- mit dem Göttlichkeitstopos in der ekphrastischen Literatur vgl. Zeitlin (2013) $22-24$. 
Über das Enkomion auf den Satyrn in seiner neuen, vegetativen Gestalt kommt Dionysos zum eigentlichen Zweck seiner Rede: dem Lob auf sich selbst als Weingott.

Zugleich mit der Einführung der Ampelos-Figur setzt der Dichter die für den Gesamtplan der Dionysiaka wesentliche Entwicklung in Gang: Dionysos wird auf seinem Weg zur Vergöttlichung im Olymp seine wichtigsten Attribute erhalten, den Weinstock und das daraus gewonnene Produkt, den Wein. Gleichzeitig damit wird er auch seinen charakteristischen Wesenszug als Gott der Trauben, des Rebensaftes und der Berauschung zugesprochen bekommen und ist so für sämtliche folgenden Ereignisse gerüstet. Der erzähltechnische Knotenpunkt der Ampelos-Episode führt vom jungen Satyrn direkt zum innersten Wesen des neuen Gottes, Ampelos wird zu einer für den Fortschritt des Epos unentbehrlichen Figur und erhält aus diesem Grund eine ausführliche Charakterisierung durch den Dichter.

\subsection{Ampelos in neuer Gestalt}

Zur Vollendung seines Wesens gelangt Ampelos erst durch die Verwandlung in seine neue Daseinsform, den Weinstock. Nach seinem gewaltsamen Tod in Nonn. D. 11,188223 erfolgt in Nonn. D. 12,173-187 die Metamorphose, allerdings erst nach der Einschaltung einer Reihe von Episoden: ${ }^{55}$

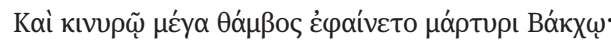

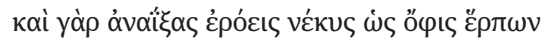

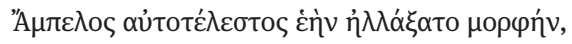
175

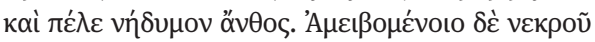

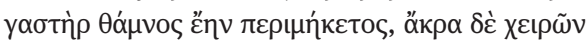

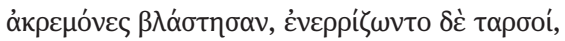

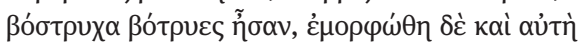

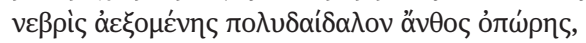

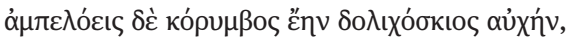

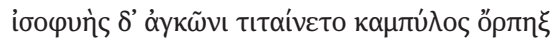

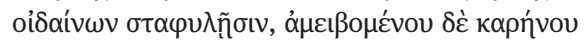

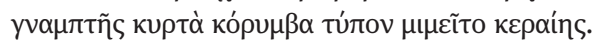

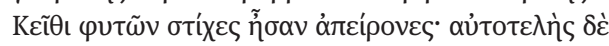

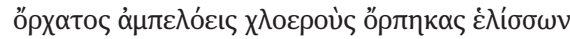

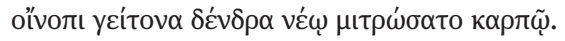

Und Bakchos wurde trauriger Zeuge eines großen Wunders: Denn der liebreizende Tote erhob sich kriechend wie eine Schlange, (175) Ampelos änderte spontan seine Gestalt und wurde zu einer Labsal bringenden Pflanze. Und der Tote verwandelte sich: Da wurde sein Bauch ein weit ausladender Strauch, an den Spitzen seiner Hände sprossen Zweige hervor, die Füße schlugen Wurzeln, die Haare wurden zu Trauben, verwandelt wurde selbst (180) die Fellbekleidung in die

55 Dies sind die Trauerreden des Dionysos (Nonn. D. 11,224-350), die Kalamos-Karpos-Erzählung (351 - 485), das Horen-Intermezzo und die Tafeln der Harmonia $(11,485$ - 12,117) sowie die Trauer der Natur (12,117-137) und die Rede der Moire Atropos (138-172). 
gesprenkelte Pflanze des reifen Spätsommers. Der lange Hals war die Spitze des Weinstocks, gleich dem Arm breitete sich ein krummer Sprössling aus, überbordend von Trauben, und als sich sein Haupt verwandelt hatte, glich es an Gestalt der gebogenen Spitze eines krummen Hornes. (185) Dort waren endlose Reihen von Pflanzen. Und der fertige Weingarten ließ seine grünlichen Sprösslinge ranken und umkränzte die benachbarten Bäume mit der neuen Weinfrucht.

Die direkte Linie, die Nonnos zwischen der vorliegenden Stelle und dem Porträt des Ampelos in Nonn. D. 10,175-192 zieht, wird durch thematische und wörtliche Entsprechungen verdeutlicht. ${ }^{56}$ Der Satyr erhält in der Verwandlungsszene ein zweites Porträt, in welchem wiederum sein Äußeres von Kopf bis Fuß beschrieben wird. Der Übergang in die neue Daseinsform wird durch direkte Bezugnahme auf die Körperteile des Satyrn illustriert: Die Haarlocken werden zu Weintrauben (Nonn. D. 12,179), das gescheckte Hirschfell nimmt die gesprenkelte Musterung der Trauben an (180). ${ }^{57}$ Ein weiteres Indiz dafür, dass der Dichter von Beginn der Ampelos-Episode an sukzessive auf deren Endpunkt hinarbeitet, liegt in der Ekphrasis des ersten Weingartens (Nonn. D. 12,185-187), an die eine weitere Metamorphose anschließt, die des Satyrn Kissos in den Efeu (188-192):

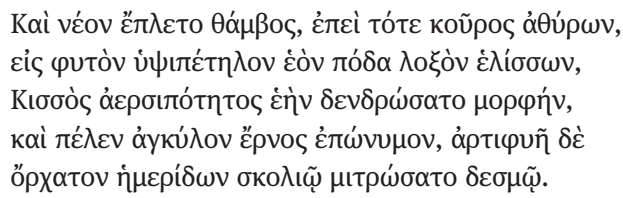

Und es begab sich erneut ein Wunder: Als gerade zu dieser Zeit ein Junge spielte, da wand er seine Füße überkreuzt um die hochbelaubte Pflanze, (190) Kissos, nach oben in die Lüfte rankend, verwandelte seine Gestalt in einen Baum und verlieh dem krummen Spross seinen Namen. Und eben geboren bekränzte er den Rebengarten mit seiner gewundenen Fessel.

Die Verwandlungsszene bietet eine Reihe von wörtlichen Entsprechungen zur unmittelbar vorangehenden Metamorphose des Ampelos und stellt ein wichtiges erzählerisches Element dar. ${ }^{58}$ Was bereits im Ampelos-Porträt angedeutet worden ist, die Verwandlung des Jünglings in eine Pflanze, wird nun zweifach realisiert. Die erste, vom Gott selbst durchgeführte Weinlese im Anschluss an die Metamorphosen der beiden Satyrn führt dem Leser den Kern der Erzählung abermals unmissverständlich vor Augen (Nonn. D. 12,193-206):

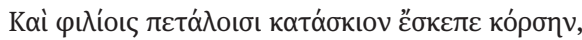

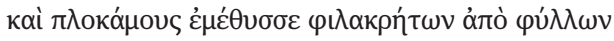

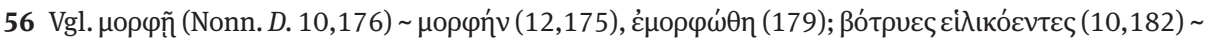

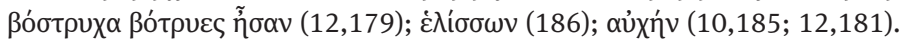

57 Vgl. Vian (1995) 196 Anm. ad 176-184.

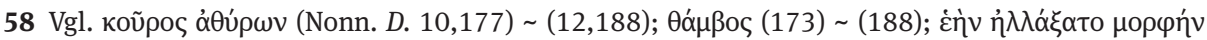

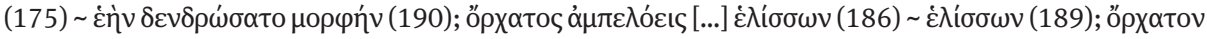

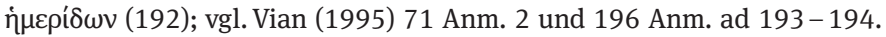




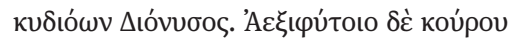

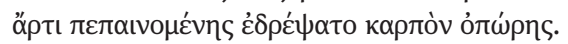

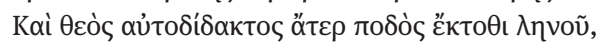

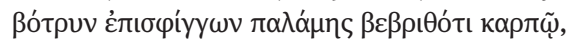

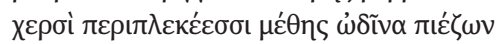

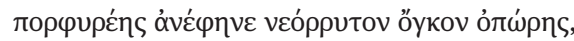

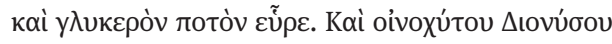

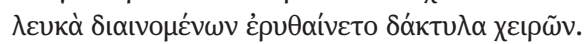

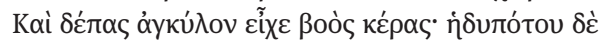

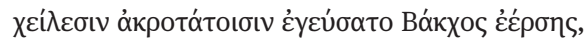

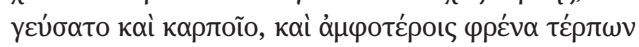

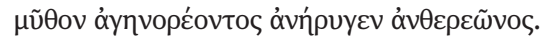

195

200

205

Und mit den lieblichen Blättern verschaffte er sich schattigen Schutz für seine Schläfe, und er machte seine Locken trunken mit Blättern, dazu bestimmt, ungemischter Wein zu sein, (195) der stolze Dionysos. Und vom pflanzennährenden Knaben erntete er die eben reif gewordene Frucht des Herbstes. Und der Gott umfasste als sein eigener Lehrer und ohne Füße und Kelter eine Weintraube von schwerer Frucht mit seiner Faust, presste mit ineinander verschränkten Händen die Früchte der Trunkenheit aus, (200) schuf so den neuen, roten Saft des Herbstes und erfand das süße Getränk. Und die weißen Finger des Dionysos wurden rot, als die Hände austeilten und er den Wein einschenkte. Als Becher hielt er das krumme Horn eines Rindes. Bakchos kostete mit spitzen Lippen vom süßen Saft, (205) er kostete auch von der Frucht, und an beidem erfreute er seine Sinne und ließ das Wort aus seinem stolzen Inneren erschallen.

Mit der Verkostung des ersten Weines verbindet sich Dionysos gleichsam physisch mit Ampelos, erhält seinen typischen Wesenszug übertragen und kann nun nach Belieben über Pflanze und Getränk verfügen. Die enge Verquickung mit seinem Hauptattribut zeigt sich noch einmal in besonderer Weise in Nonn. D. 36,307-312, wo er sich selbst in einen Weinstock verwandelt, um sich gegen den Inder Deriades zur Wehr zu setzen. ${ }^{59}$ Aus der erzählerischen Konzeption der Ampelos-Episode wird deutlich, dass Nonnos nicht beim bloßen Porträt des Satyrn stehen bleibt, sondern auf die Genese des Weines als den Höhepunkt der Erzählung hinarbeitet. Erst mit dem Tod seines Lieblingssatyrn erhält Dionysos sein ureigenstes Wesen zugeschrieben, und erst mit der Wiederauferstehung des Ampelos verfügt er über seine effektivsten Waffen, Weinstock und Wein.

\subsection{Ampelos in den Dionysiaka außerhalb der Ampelos-Episode}

In Kontrast zum besonderen Stellenwert, den die Episode innerhalb der Gesamtkomposition der Dionysiaka einnimmt, steht die relativ seltene Namensnennung des Ampelos in den übrigen Büchern der Dionysiaka. Der Satyr wird insgesamt 38-mal im Epos genannt, davon allein 36-mal in der Ampelos-Episode. ${ }^{60}$ Die zweimalige Nen-

59 Zu den wörtlichen Parallelen zu Nonn. D. 12,176 - 184 siehe Vian (1995) 196 Anm. ad 176-184. 60 Der Nominativ 'A $\mu \pi \varepsilon \lambda$ oৎ findet sich 16-mal (Nonn. D. 10,178, 239, 286, 317, 401, 413, 416, 424; 11,18, 25, 44, 57, 277; 12,102, 145, 175), der Akkusativ 'A $\mu \pi \varepsilon \lambda$ ov 14-mal (10,263, 353; 11,53, 81, 84, 
nung außerhalb der Bücher 10, 11 und 12 erfolgt in einiger Distanz zur Ampelos-Episode: Am Beginn seiner Rede im 27. Buch ruft Zeus Apoll um Unterstützung im Kampf gegen die Inder auf, indem er an das verwandtschaftliche Verhältnis zwischen Apoll und Dionysos sowie an die enge kultische Beziehung zwischen den beiden appelliert

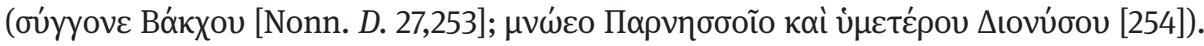
In diesem Zusammenhang kommt Zeus auch auf Ampelos zu sprechen (Nonn. $D$. 27,255f.):

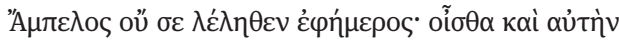

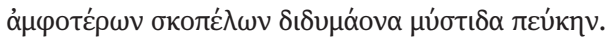

(255) Ampelos, der nur kurz das Tageslicht sah, blieb vor dir nicht verborgen. Und du kennst dieselbe mystische Doppelfackel beider Berggipfel. ${ }^{61}$

Die beiden Verse thematisieren das Schicksal des Ampelos und bringen zudem den dionysischen Kult ins Spiel. An die Reminiszenz von Ampelos' Kurzlebigkeit ( $\dot{\varepsilon} \varphi \eta^{-}$ $\mu \varepsilon \rho o \varsigma$ [Nonn. D. 27,255]) ${ }^{62}$ sind dionysische Kultsymbole geknüpft, wodurch eine Abhängigkeit des dionysischen Weinkultes vom Satyrn hergestellt und Ampelos' Tod zu einer Vorbedingung für den Weinkult wird. Als Argument, um Apoll zum Einlenken in der aktuellen Situation zu bewegen, dient Zeus der tragische Tod des Ampelos, der beim delphischen Gott ein Verbundenheitsgefühl hervorrufen soll, da dieser, wie die mythologische Tradition lehrt, durch den ebenfalls frühzeitigen Tod seines geliebten Hyakinthos einen ähnlichen Verlust hat hinnehmen müssen. ${ }^{63}$ Beim lediglich vage angedeuteten Mythos handelt es sich um die Wiederaufnahme eines in der AmpelosEpisode mehrfach angewandten mythischen Exempels, das dem Schicksal des Ampelos das des jungen Hyakinthos zur Seite stellt. ${ }^{64}$

In der zweiten Passage, in der Ampelos namentlich genannt wird, erfolgt abermals eine Anspielung auf Ampelos' Todesschicksal, und zwar in der Geschichte um Hymenaios, der ähnlich wie der Satyr zuvor als Geliebter des Dionysos genannt wird: Hymenaios wird durch den Pfeilschuss des Melaneus schwer verwundet, woraufhin Dionysos eine Trauerrede hält (Nonn. D. 29,108-150), in der Hymenaios dem Ampelos

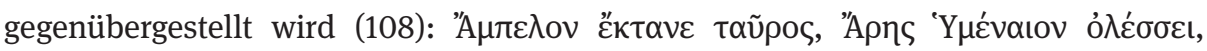

116, 136, 190, 225, 306, 316, 327; 12,153, 250) und der Vokativ "А $\mu \pi \varepsilon \lambda \varepsilon$ sechsmal $(11,10,39 ;$ 12,167, 207, 212, 270).

61 Bei den Berggipfeln handelt es sich um die Phaidriaden nahe Delphi, bei den Fackeln um die mystischen Fackeln, die bereits in Nonn. D. 9,286 genannt werden (бùv áypúrvoı dort im Rahmen von Reigentänzen brennen, die Ino dem noch jungen Dionysos stiftet (9,283-289), vgl. Agosti (2004) 235 Anm. 256.

62 'Е

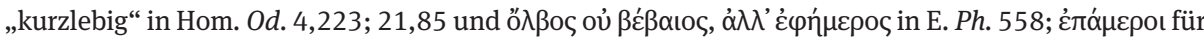
die kurzlebigen Menschen in Pi. P. 8,95; A. Pr. 83; siehe auch Pi. Fr. 157. - Zu den hapax legomena in den Dionysiaka siehe Espinar Ojeda (2002).

63 Vgl. Agosti (2004) 234 Anm. 255.

64 Siehe dazu Kap. 4.3. 
„Ampelos tötete der Stier, Ares wird Hymenaios zugrunde richten“. Dass dieses postulierte parallele Schicksal jedoch keineswegs eintritt, sondern Dionysos’ Aussage der rhetorischen Dramatisierung des Augenblicks dient, ergibt sich aus dem folgenden Geschehen: Anstatt dass Hymenaios das Todesschicksal ereilt, gelingt es dem Weingott durch seine medizinischen Fähigkeiten, seinen Gefährten zu heilen (Nonn. $D$.

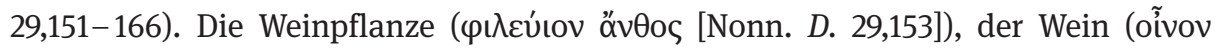

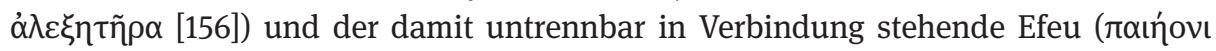

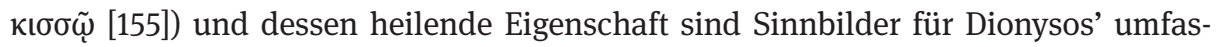
sende göttliche Wirkkraft, die Metamorphose des Ampelos in den Weinstock bildet so die Voraussetzung für die Genesung des Hymenaios.

An den beiden genannten Stellen stellt der Dichter die für ihn wesentlichen Aspekte, Ziele und Endpunkte der Ampelos-Episode heraus: Ampelos' Todesschicksal

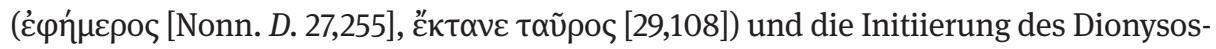
Kultes, die eine Folge der Metamorphose des Satyrn in den Weinstock darstellt. Ist beides, Tod und Wiederauferstehung in neuer Erscheinungsform, erreicht, hat auch Ampelos seinen narrativen Zweck erfüllt. Der Satyr verschwindet nach dem Ende der ihm gewidmeten Episode beinahe vollständig aus der Erzählung, weicht dem mit Rebstock, Wein und Efeu ausgestatteten Dionysos und geht vollständig im neuen Attribut seines Gottes auf.

Reminiszenzen an Ampelos' Schicksal finden sich jedoch nicht nur in den Bü-

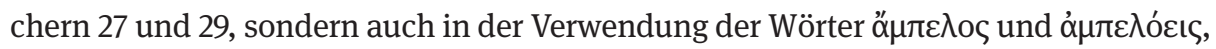
die der Dichter Nonnos im Hinblick auf die Komposition seiner Entstehungsgeschichte des Weines funktionalisiert. Das Substantiv å $\mu \pi \varepsilon \lambda$ os in der Bedeutung „Weinstock“ findet sich in den Dionysiaka selten, insgesamt nur achtmal, davon siebenmal nach Abschluss der Ampelos-Episode, also nachdem der Weinstock durch den Tod und die Metamorphose des Ampelos in die Welt gekommen ist und nun ein charakteristisches Wesensmerkmal des Gottes Dionysos darstellt. ${ }^{65}$ Von diesen sieben Textstellen seien zwei herausgegriffen: Am Ende des 16. Buches irrt die Nymphe Nikaia nach ihrer Verbindung mit Dionysos auf der Suche nach dem Gott, an dem sie sich rächen will,

\footnotetext{
65 (1.) Nikaia will Weinstöcke vernichten (Nonn. D. 16,378); (2.) Hera befürchtet, der Wein des Dionysos könnte für die Götter schädlich sein $(31,239)$; (3.) Dionysos setzt Weinranken als Waffen im

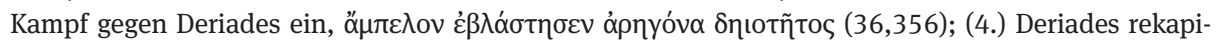
tuliert in seiner Feldherrenrede die Sukzession Zeus - Zagreus - Deriades und nennt den Weinstock ein

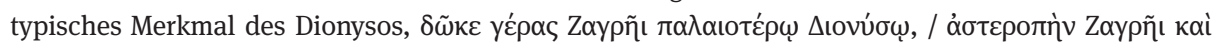

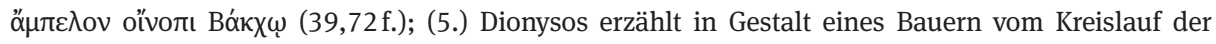
Jahreszeiten, u. a. vom Herbst mit den reifen Trauben und dem zur Ernte bereiten Getreide, ä $\mu \pi \varepsilon \lambda$ o

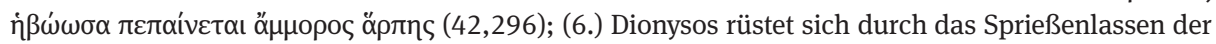

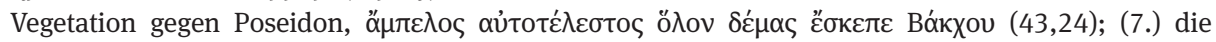

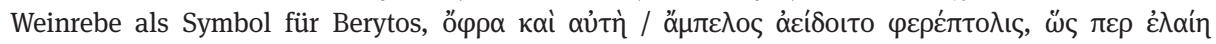

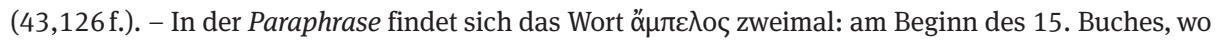

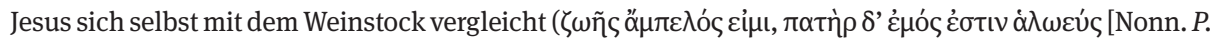

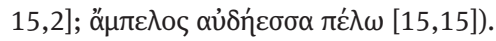


durch die Berge und ist gewillt, jeden Weinstock, den sie findet, zu zerstören (Nonn. $D$. 16,374-378):

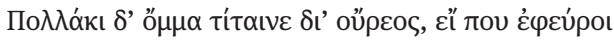

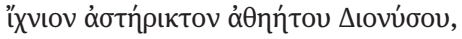
375

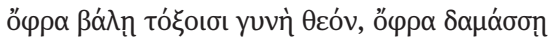

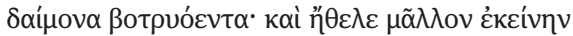

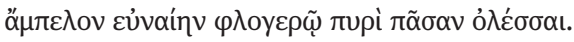

Und oft ließ sie den Blick über die Berge schweifen, ob sie irgendwo (375) eine frische Spur des unsichtbaren Dionysos fände, damit sie ihn mit Pfeilen treffen könne, sie, eine Frau einen Gott, damit sie die traubenreiche Gottheit bezwinge. Und noch lieber wollte sie jenen Weinstock in ihrem Brautbett als Ganzen mit flammendem Feuer vernichten.

Dionysos wird hier als Gott des Weines, der Wein als der Gott selbst gesehen, ganz

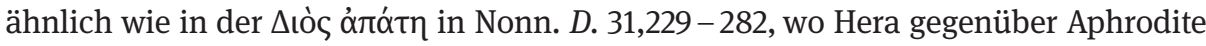
dem neuen Gott Dionysos und der für ihn vorgesehenen Herrschaft im Olymp eine klare Absage erteilt. Aus Furcht davor, dass Zeus und sie selbst ihre angestammte Macht einbüßen könnten, äußert Hera einen భóyos gegen den Weinstock (Nonn. $D$. 31,238f.):

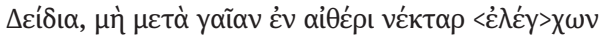

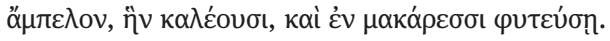

Ich fürchte, dass er [sc. Zeus] nach der Erde auch im Himmel den Nektar schmäht und den Weinstock, wie sie ihn nennen, auch unter den Glückseligen wachsen lässt.

Der Grundtenor in den beiden Textstellen ist derselbe: Der neu in die Welt gekommene Weinstock steht mit Dionysos in Verbindung und wird zum Sinnbild des neuen Gottes. Auch in den übrigen Passagen, die den Weinstock anführen, dient dieser entweder als unfehlbare und wirkungsmächtige Waffe gegen die Inder (Nonn. D. 36,356; 43,24) oder ist Symbol für Dionysos und seinen Wirkungsbereich (39,72f.; 42,296; 43,126f.). Nicht zufällig finden sich alle sieben Textstellen, in denen das Substantiv ő $\mu \pi \varepsilon \lambda$ o genannt wird, nach der Ampelos-Episode, die Existenz der Pflanze ist somit die Konsequenz der Ereignisse der Bücher 10-12.

Lediglich ein einziges Mal wird das Wort ö $\mu \pi \varepsilon \lambda$ os vor der Ampelos-Episode verwendet: In einer Rede an Aion, die Musen und die Horen prophezeit Zeus die Ankunft des Weines, des Sorgenlösers für die Menschheit (Nonn. D. 7,73-105):

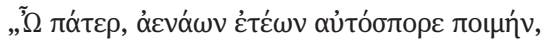

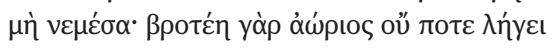

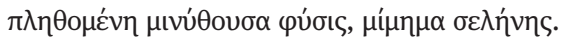

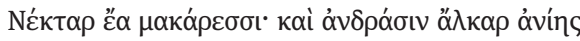

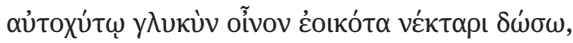

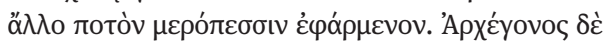

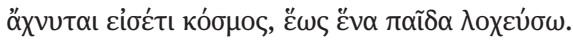

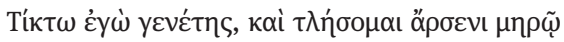

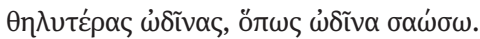




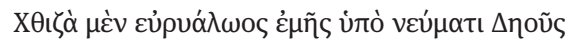

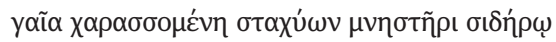

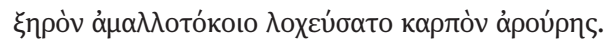

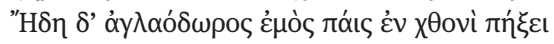

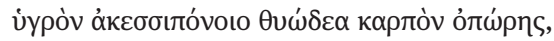

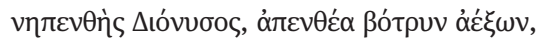

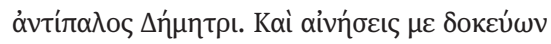

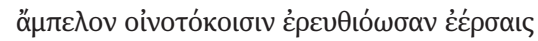

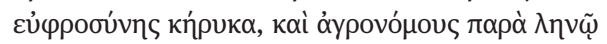

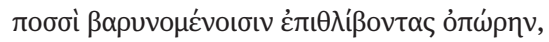

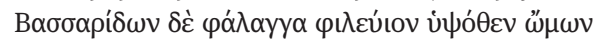

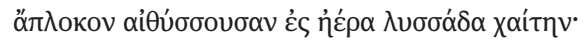

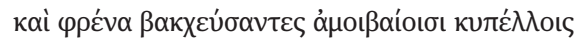

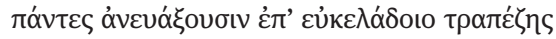

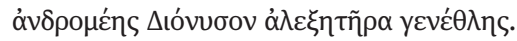

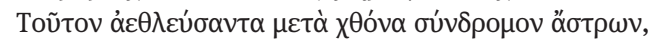

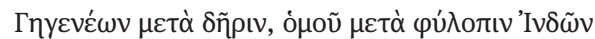

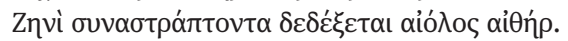

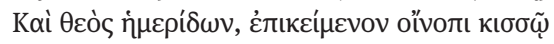

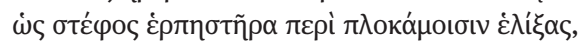

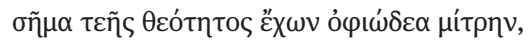

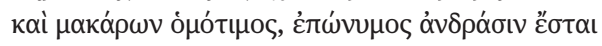

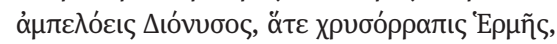

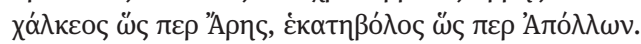

„Vater, selbst gezeugter Hüter der immerwährenden Jahre, zürne nicht! Denn niemals hört das sterbliche und unzeitige Geschlecht auf (75) zur Vollendung zu gelangen und wieder zu vergehen, ein Abbild der Selene. Den Nektar überlass' den Glückseligen! Ich werde den Menschen zur Abwehr des Schmerzes den süßen Wein geben, der ähnlich dem von selbst fließenden Nektar ist, ein anderes Getränk, das für die Menschen passt. Der uranfängliche Kosmos ist solange vor Kummer betrübt, bis ich einen Sohn zur Welt bringe. (80) Ich werde gebären, ich, der Vater, und ich werde in männlichem Schenkel weibliche Wehen erdulden, um das Ungeborene zu retten. Gestern wurde die Erde auf einen Wink meiner Deo, der mit den weiten Tennen, vom werbenden Eisen für die Ähren zerfurcht und brachte trockene Frucht am garbenbringenden Feld hervor. (85) Bald wird mein Sohn, glänzende Geschenke bereitend, in die Erde die feuchte, duftende Frucht der schmerzlindernden Traube einsetzen, der sorgenbannende Dionysos, indem er die sorgenlösende Weintraube wachsen lässt, ein Gegenstück zu Demeter. Und loben wirst du mich, wenn du erkennst, dass der Weinstock sich von Wein hervorbringenden Tropfen rötet, (90) der Verkünder der Glückseligkeit, dass die Landleute bei der Kelter mit schweren Füßen die Traube auspressen und die jauchzende Schar der Bassariden von den Schultern herab die ungeflochtene, wirre Mähne in die Luft schüttelt. Und rasend im Sinn durch die gegenseitig gereichten Becher (95) werden alle am zünftig lärmenden Tisch Dionysos bejubeln, den Helfer des Menschengeschlechts. Diesen wird, wenn er nach der Erde auch mit der Versammlung der Sterne gerungen hat, nach dem Kampf mit den Giganten, wie auch nach der Schlacht mit den Indern, der gleißende Äther aufnehmen, und er wird zusammen mit Zeus Blitze entsenden. (100) Und der Gott der veredelten Weinstöcke wird sich ein Gewinde aus Weinlaub wie einen Kranz um die Locken legen und als Zeichen seiner Göttlichkeit eine schlangenartige Binde haben. Und Seligen gleichend an Ehren wird er bei den Menschen mit einem Beinamen genannt werden: ,Dionysos mit dem Weinlaub', wie ,Hermes mit dem goldenen Stab', (105) ebenso wie ,der eherne Ares‘ und wie ,der Ferntreffer Apoll‘.“ 


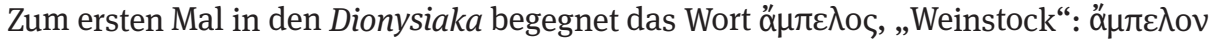

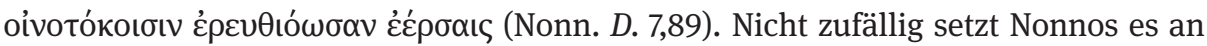
diese Stelle, mittels der gezielten Positionierung des Substantivs gelingt es ihm, die Ampelos-Episode vorzubereiten und somit schlüssig in die Gesamtkomposition des Epos einzufügen. Das von Zeus vorhergesagte Kommen der Weinpflanze ist ein Vorverweis auf die Geburt des Dionysos und dessen Ausstattung mit seinem wichtigsten Attribut. Ampelos und sein Schicksal werden nirgendwo genannt, der Schwerpunkt liegt auf dem Resultat der Bücher 10 -12, der Genese des Weinstocks und dem ersten Keltervorgang. Mit der Entstehung des Weines erfolgt ein wesentlicher Schritt in Richtung der Erfüllung des epischen Plans. Allein durch dieses Attribut wird Dionysos imstande sein, die an ihn gestellten Aufgaben zu bewältigen, nur so wird er in den Olymp einziehen können.

Der Zustand des Kosmos nach der Bildung des Weinstocks wird in der Zeus-Rede insofern antizipiert, als nicht nur das Wort ő $\mu \pi \varepsilon \lambda$ os zum ersten Mal in Erscheinung tritt, sondern sich ein ganzes Wortfeld um den Themenkreis Wein auftut und Nonnos

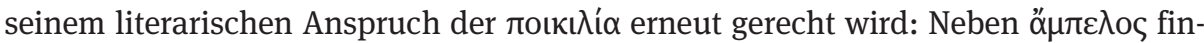

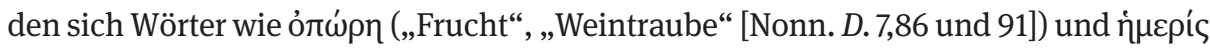
(,veredelte Weinrebe“ [100]); Weinbeere, Weintraube, Saft und Kelter begegnen zudem

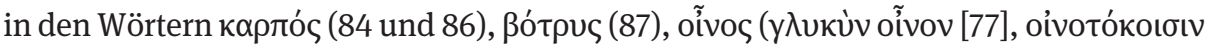

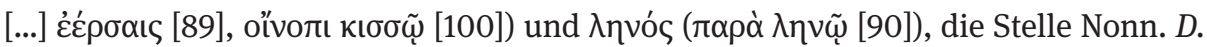
7,100 bringt außerdem den symbiotisch mit der Weinrebe vereinten Efeu. Mit der ZeusRede arbeitet Nonnos kontinuierlich auf einen der Höhepunkte seines Epos hin, die Ausstattung des neuen Gottes mit seinem wichtigsten Attribut sowie die Stiftung seines Kultes. Dabei gelingt es dem Dichter, einen Spannungsbogen aufzubauen, indem er das Kommen des Weines nicht nur vorläufig aufschiebt - es vergehen noch dreieinhalb Bücher bis zum Beginn der Ampelos-Episode -, sondern die genauen Umstände dieser Genese noch im Unklaren lässt. Festgehalten werden $\mathrm{zu}$ diesem frühen Zeitpunkt lediglich Höhepunkt und Resultat der göttlichen Bemühungen.

Die Zeus-Rede verwendet erstmals in den Dionysiaka auch das Epitheton $\alpha \mu \pi \varepsilon-$

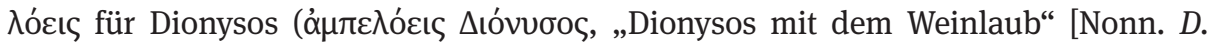
7,104]). Auch dieses Adjektiv wird vom Dichter sparsam und gezielt eingesetzt, insgesamt nur dreimal vor der Ampelos-Episode, das erste Mal überhaupt in den Dionysiaka an der genannten Stelle der Zeus-Rede, sodann unmittelbar danach, wenn Eros den Liebespfeil auswählt, der für Zeus und Semele vorgesehen ist (Nonn. D. 7,129135):

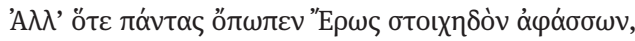

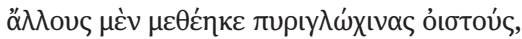

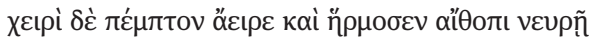

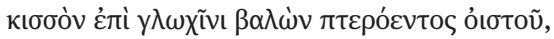

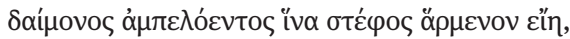

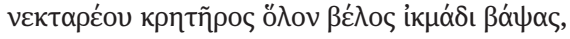

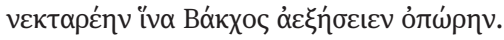




\begin{abstract}
Aber als Eros alle angesehen und einen nach dem anderen geprüft hatte, (130) da legte er die anderen Pfeile mit feuriger Spitze zur Seite, hob mit der Hand den fünften hoch, passte ihn in die glänzende Sehne ein und tat Efeu auf die Spitze des gefiederten Pfeils, damit der Gottheit mit dem Weinlaub ein Kranz angeheftet werde. Und er tauchte das ganze Geschoss in die Flüssigkeit eines Kraters mit Nektar, (135) damit Bakchos die Weintraube ähnlich dem Nektar wachsen lasse.
\end{abstract}

Das Bestreichen des Pfeiles mit Nektar, dem göttlichen Abbild des Weines, nimmt Dionysos' göttlichen Status vorweg. Dionysos, dessen Existenz zu diesem Zeitpunkt erst durch einen göttlichen Plan festgesetzt ist, wird von Anfang an mit seinen typi-

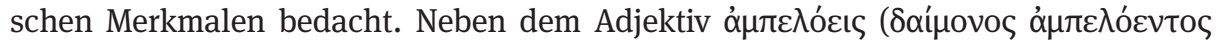
[Nonn. D. 7,133]) findet sich das aus der Zeus-Rede bekannte Wortmaterial zum The-

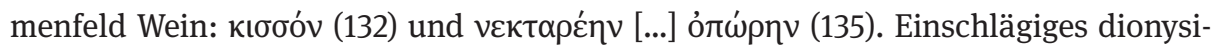
sches Vokabular ist zudem an zwei weiteren Schlüsselstellen der Dionysos-Biographie zu beobachten: Bei der ersten Stelle handelt es sich um den ícò ` yó $\mu$ o son Zeus und Semele am Ende des 7. Buches, der in eine unverkennbar dionysische Atmosphäre gehüllt ist; der Göttervater, ganz in dionysischer Aufmachung, zeigt sich in sämtlichen Erscheinungsformen, in die sich später auch Dionysos verwandeln kann, wobei sich

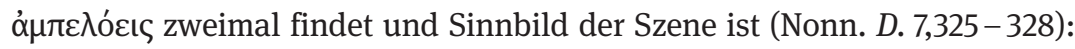

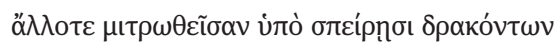
325

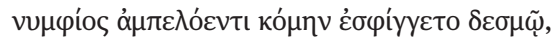

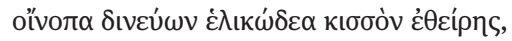

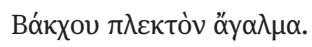

(325) Außerdem band er das Haar, das von Schlangenwindungen umwunden war, wie ein Bräutigam mit einem Reif aus Weinlaub zusammen und drehte spiralförmig den weinfarbenen Efeu in die Locken hinein, den geflochtenen Schmuck des Bakchos.

Neben $\alpha$ $\mu \pi \varepsilon \lambda$ ócıৎ erscheint auch hier wieder der Efeu, und zwar in Verbindung mit dem

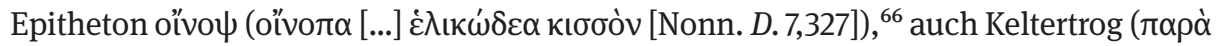

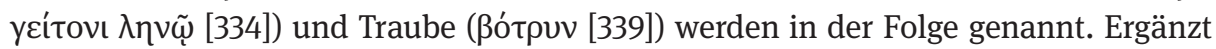
wird das Bild durch das bereits in der Zeus-Rede zu findende Adjektiv $\varphi$ ı $\lambda$ v́ıov (Nonn. D. 7,92 und 335), das in Nonn. D. 7,335 noch besonders herausgehoben wird, wenn im selben Vers auch das Simplex củıv gesetzt ist. Die Weinrebe, die in der Rede des Göttervaters eindeutig vom altbewährten göttlichen Nektar geschieden ist (Nonn. $D$. 7,76-78), wird genauso wie an der Stelle mit Eros und seinem Pfeil (135) mit der

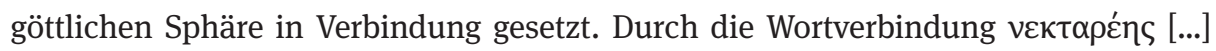
ỏ $\omega$ pns (338) wird das neue Getränk erhöht und erhält gleichsam göttlichen Status. Als Abschluss der Szene um Zeus und Semele taucht das bereits aus der Passage mit

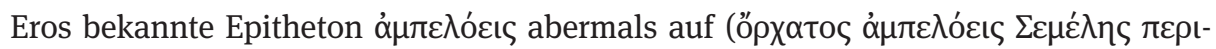

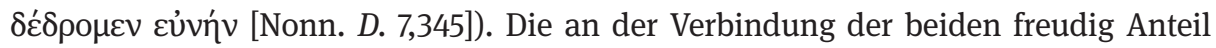

66 O"vo $\psi$ und кıббóৎ werden wenige Verse später bei der Beschreibung des Thyrsos erneut begegnen

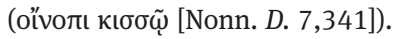


nehmende Natur lässt hier einen ganzen Garten von Weinstöcken sprießen, obwohl die eigentliche Genese der Pflanze noch nicht vollzogen ist: Nonnos kommt es dabei keineswegs auf den exakten (chrono)logischen Zusammenhang an, sondern auf die dionysische Stimmung, mit der die Episode um Zeus und Semele ausgestattet werden soll.

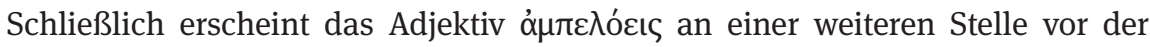
Ampelos-Episode: Im 9. Buch macht die Amme Mystis das Kleinkind Dionysos mit dem Instrumentarium des bakchischen Kultes vertraut (Nonn. D. 9,111-131). ${ }^{67}$ Neben einem

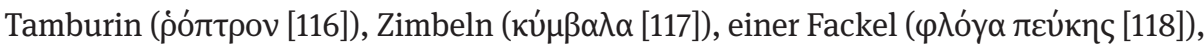

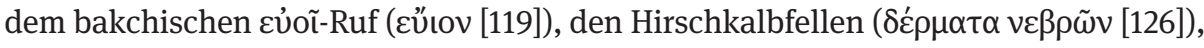

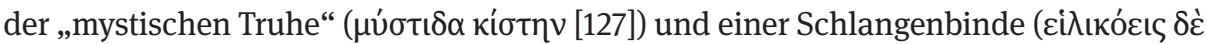

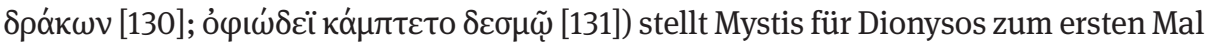
auch Efeutrauben (кó $\rho \mu \beta$ os [120 und 123]) ${ }^{68}$ und Weinranken zur Verfügung (120 124):

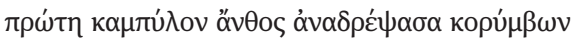

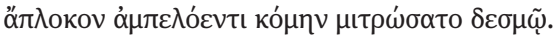

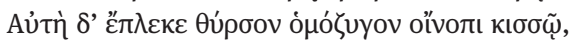

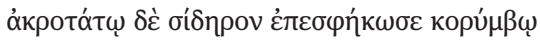

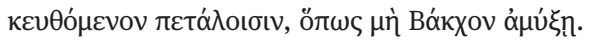

(120) Zum ersten Mal pflückte sie die gekrümmte Blüte von Efeutrauben und umwand das ungeflochtene Haar mit dem Band aus Weinlaub. Sie selbst flocht einen Thyrsos, zusammengebunden mit weinfarbenem Efeu, und band ein Eisen an die Spitze des Efeubüschel, verdeckt von Blättern, damit es Bakchos nicht steche.

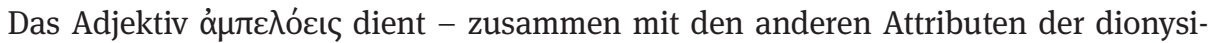
schen Sphäre - gleichsam als Siegel und Marke für die wichtigsten Stationen auf dem Weg, den Dionysos zur Erlangung seines vollwertigen Status zurücklegen muss. Die erste Station bildet die Prophezeiung der Geburt des Gottes durch Zeus im 7. Buch, gefolgt von der Episode aus seinen Kindheitstagen um die Erzieherin Mystis und die erstmalige Konfrontation des Dionysos mit seinem eigenen Kult. Nonnos setzt dieses Adjektiv gezielt im Hinblick auf die Konzeption seines Epos ein, es taucht ab Buch 11 regelmäßig auf und wird für unterschiedliche Figuren und Sachverhalte verwendet, denen allen die Zugehörigkeit zum dionysischen Einflussbereich gemeinsam ist. Mit

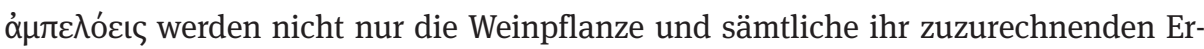
scheinungsformen, Merkmale und Funktionsbereiche charakterisiert, sondern auch

67 Zur Mystis-Szene siehe García-Gasco (2014).

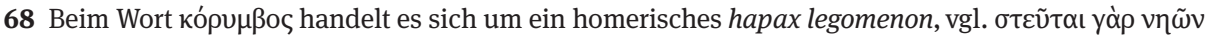

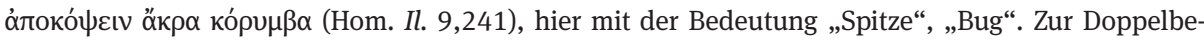
deutung des Wortes, das auch „Efeutrauben“ bedeuten kann, siehe den Eintrag kappa, 991 im Lexikon

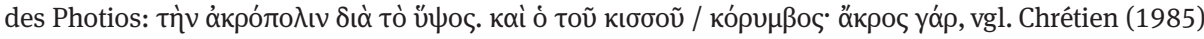
110. 
das dionysische Personal, vor allem aber Dionysos selbst. ${ }^{69}$ Nonnos richtet seine etymologischen Wortspiele auf die erzähltechnische Konzeption seiner Geschichte aus, indem er die Bedeutung des Namens Ampelos mit seinem Träger verknüpft. Der Name charakterisiert nicht nur den Satyrn, sondern auch den Gott Dionysos, in diesem einen Wort ist die gesamte Narration enthalten und wird gleichsam vorweggenommen. $^{70}$

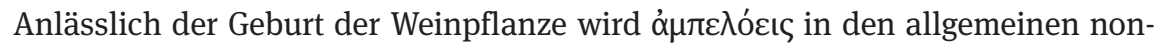
nianischen Wortschatz aufgenommen und regelmäßig verwendet. Die wenigen Male, die Nonnos das Adjektiv vor der Ampelos-Episode einsetzt, sind vom Dichter bewusst gewählt, um gezielt auf den Höhepunkt der Erzählung hinzuarbeiten. Durch den

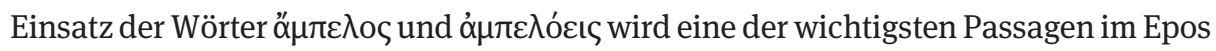
vorbereitet: die Metamorphose des Ampelos und die Entstehung des Weines. ${ }^{71}$ Dieses Ereignis bildet die Voraussetzung für alle weiteren Stationen auf Dionysos' Weg in den Olymp, der Mythos Ampelos wird im Spannungsfeld zwischen Leben und Tod angesiedelt. Die neue Pflanze verfügt über Charakteristika, die auf das tragische Schicksal der Satyr-Figur zurückverweisen, die Verwandlung in Pflanze und Getränk ermöglicht

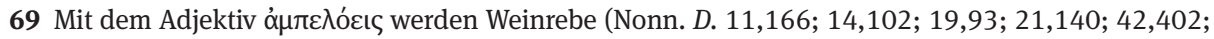
47,71), Weintrauben $(11,516 ; 12,102 ;$ 45,146), Efeu $(12,181,292,317 ; 17,263,352 ; 19,130 ; 20,296$; 27,206; 30,199; 35,339, 360), Garten (12,186; 42,278, 522), Hecke (16,272), Gärtner (43,85), Schössling (21,29; 24,32f.; 44,241), Wein (14,255; 18,125; 19,56), der Sirius $(43,171)$ und verschiedene Gegenstände wie Kopfbinde $(29,267 ; 37,314 ; 47,525)$, Haare $(36,156)$, ein Geschenk $(41,6)$, Geruch $(25,297)$, ein Seil $(18,188)$ und der Himmel $(31,242)$ bedacht, ebenso wie Wörter des

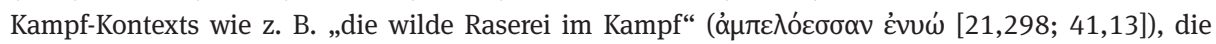

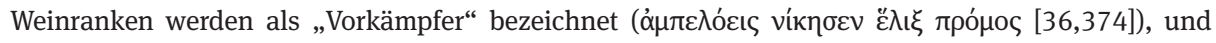
Wurfgeschosse sind mit Weinranken versehen (14,354, 394; 17,296; 29,231; 30,259; 43,322; 45,14). Das Adjektiv erhalten auch einzelne Figuren: Ambrosia (21,39 und 53) und Gigarto (21,77), mit Ab-

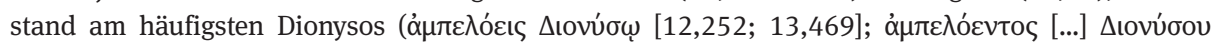

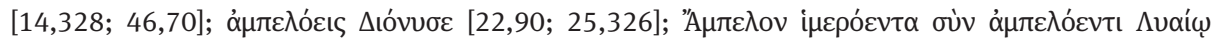

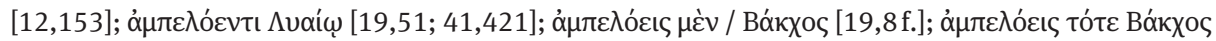

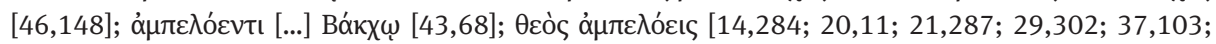

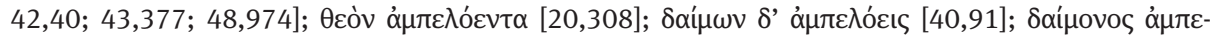

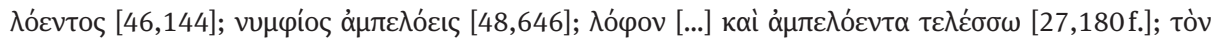

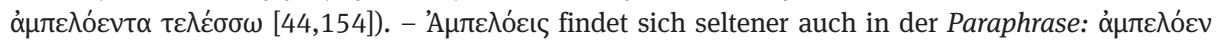

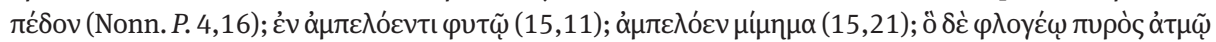

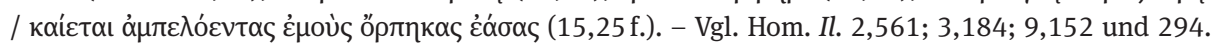
70 Zur narrativen Funktion von Etymologien vgl. Tsitsibakou-Vasalos (2007) 6: „These techniques, in addition to their serviceability as mnemonic aids in an orally composed and performed poetry, also allow poetic etymology to emerge as a narrative factor that pulls together the strings of the story and promotes its deployment. Etymology targets the lexical and thematic unification of the poetic work“; vgl. auch Skempis u. Ziogas (2009) 213-215, hier: 214: „Etymology is compressed narrative, and unpacking the etymological potential of a word is to deploy its narrative force (vis).“

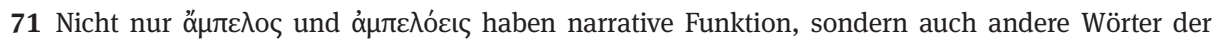
dionysischen Sphäre, die ebenfalls nur selten vor der Ampelos-Episode zu finden sind: ßótpus (Nonn. D. 1,33 und 528; 7,87 und 339; außerdem lediglich einmal in der Paraphrase, vgl. Nonn. P. 15,3),

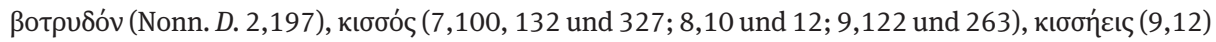

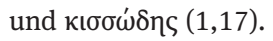


es dem Jüngling, über seinen Tod hinaus Geliebter des Gottes zu bleiben. ${ }^{72}$ Tod und Auferstehung des Satyrn spiegeln zudem konstituierende Elemente dionysischen Kultes: So wie Ampelos stirbt und wiederaufersteht, so wie die Trauben sich durch Keltern zu ihrer neuen Erscheinungsform, dem Wein, wandeln, so erlangt DionysosZagreus in Gestalt des Dionysos-Bakchos erneut das Leben. Die Wiedergeburt des Ampelos steht für die Wiederauferstehung des Dionysos sowie allgemein für den Sieg des Lebens über den Tod. ${ }^{73}$

Um seiner Ampelos-Figur stärkeres Profil zu verleihen und deren Schicksal auszugestalten, greift Nonnos zudem auf die narrative Technik der Synkrisis zurück, die dem rhetorischen Standardrepertoire zuzurechnen ist und in den antiken Hand- und Übungsbüchern zur Rhetorik ausführlich erläutert wird. Der Dichter macht sich bekannte Jünglinge und Götterlieblinge aus der Mythologie zunutze und diese durch vergleichende Gegenüberstellung zu einem Charakterspiegel für seinen Ampelos. 